

\section{MÉLANGES de l'Université Saint-Joseph}

Volume LXV - 2013-2014

Résumés/Abstracts

ÉTHIQUE, POLITIQUE ET RHÉTORIQUE DANS LES TRAITÉS ET LEURS COMMENTAIRES, DE L'ANTIQUité À LA RENAISSANCE. ORIENT ET OCCIDENT

Actes du séminaire tenu en 2012, UPR 76 (CNRS, Paris)

Présentation

Frédérique WOERTHER

Du neuf sur l'Alexandros chrétien?

Pierre CHIRON

Ethical and Political Commentary on Aristotle's Rhetoric

During the Renaissance

Lawrence D. GREEN

Moral Virtue in Late Antique Platonism.

Some Elements of a Background to Ethics in Early Arabic Philosophy Dominic O'Meara

Rhetoric Across Cultures: The Case of Themistius

John W. WATT

Alfarabi (870-950): Reason, Revelation, and Politics

Charles E. ButTerworth

The Influence of the Nicomachean Ethics on Medieval Jewish Thought Steven HARVEY 
Le commentaire sur l'Éthique à Nicomaque dans l'Occident latin.

Un genre, une pratique ( $\mathrm{XIII}^{\mathrm{e}}-\mathrm{XV}^{\mathrm{e}}$ siècle)

Bénédicte SÈrE

Scientia moralis and prudentia.

The Medieval Roots of a Contemporary Problem

Alexander FIDORA

*

Al-Fārābī's Introduction to the Five Rational Arts with Reference to

The Five Aphorisms (al-Fușūl al-Khamsa) and Ibn Bājja's Comments (Ta 'ālīq)

Terence J. KLEVEN

Un fragment retrouvé (sur la composition des éléments) du troisième Livre perdu du Grand commentaire au De Caelo par Averroès

Teymour Morel et Maroun AouAD

Culture vs. Civilization

Ibn Khaldūn's Muqaddima and the Literal Meaning of 'Umrān

Waseem EL-RAYES

Inventaire des marques lapidaires médiévales du Liban

Patricia ANTAKI-MAsson

OBJETS ET DOCUMENTS DIVERS PROVENANT DES FOUILLES DE JEAN MÉcÉRIAN DANS L'ANTIOCHÈnE (1932-1939)

Avant-propos

Lévon Nordiguian

Présentation

Lévon Nordiguian et Tomasz WALISZEWSKI

Le père Jean Mécérian (1888-1965)

Bibliographie sélective du père Jean Mécérian (1888-1965) 
Le Sanctuaire de Saint-Syméon-Stylite-le-Jeune au Mont Admirable à la lumière de la documentation photographique du père Jean Mécérian Mariusz GwIAZDA

Dix siècles d'une collection levantine.

Les sculptures de la collection du père Jean Mécérian

Dagmara WielgosZ-RonDOLINO

Lampes à huile de la collection du père Jean Mécérian

Tomasz WALISZEWSKI

Objets métalliques de la collection du père Jean Mécérian

Agnieszka SzULC-KaJAK

An Overview of the Medieval Pottery From the Antiochene Region

Excavated by Jean Mécérian

Tasha Vorderstrasse

Les miscellanea de la collection du père Jean Mécérian

Mariusz GwIAZDA

Nous remercions vivement Bassam Kahwagi, Roupène Basmadjian, Harouth Bezdjian, Christia Sayegh et Karam Hoyek pour avoir gracieusement mis à notre disposition leur temps et leur talent pour les retouches, le calibrage, le détourage et le scannage des illustrations reproduites dans ce volume. (E.G.) 


\title{
Les miscellanea de la collection du père Jean Mécérian*
}

\author{
Mariusz GwiAzDA
}

\section{INTRODUCTION}

Le présent article a pour objectif de présenter les petits objets archéologiques mis au jour par le père Jean Mécérian et conservés actuellement à l’Université Saint-Joseph de Beyrouth. À la veille de la seconde guerre mondiale, ce chercheur procéda à une prospection de surface de la région entre l'antique Antioche sur le fleuve Oronte et Séleucie de Piérie et réalisa des travaux archéologiques sur le site de l'église de l'Arbre de la Vie à la Montagne Noire et sur celui du monastère de Saint-SyméonStylite-le-Jeune au Mont Admirable ${ }^{1}$. Le matériel archéologique recueilli lors de ces fouilles fut transféré à Beyrouth, mais ne fit jamais l'objet de publication. Le fragment de la collection que nous présentons pour la première fois comprend: des

\footnotetext{
Je tiens à remercier M. Lévon Nordigiuan, conservateur de la photothèque de la Bibliothèque Orientale de l'Université Saint-Joseph de Beyrouth, pour m'avoir autorisé à étudier les objets archéologiques qui font l'objet du présent article, ainsi que pour son aide bienveillante pendant mes recherches menées au Musée de Préhistoire Libanaise dont il est directeur. J'exprime aussi ma très profonde gratitude à Tomasz Waliszewski, qui m'a proposé de participer au projet de publication de la collection de Jean Mécérian, et à Krzysztof Domżalski, qui a bien voulu lire le texte et me faire part de ses précieuses remarques. Mes remerciements vont également à Magda Makowska et à Andżelika Dłuska, qui ont réalisé les dessins, ainsi qu'à Bartek Wójcik, auteur des photographies.

1 Sur les travaux archéologiques de Jean Mécérian, voir: Millet G. (1933), «La mission archéologique du P. Mécérian dans 1'Antiochène », Comptes rendus des Séances de l'Académie des Inscriptions et Belles-Lettres 77/3, p. 343-348; id. (1935), «Un rapport du R.P. Mécérian sur les fouilles au monastère de Saint-Syméon-le Jeune au Mont Admirable (Syrie)», Comptes rendus des Séances de l'Académie des Inscriptions et Belles-Lettres 79/2, p. 195-197; id. (1936), « Rapport du P. Mécérian sur les fouilles au monastère de Saint-Syméon le Jeune au Mont Admirable (Syrie) », Comptes rendus des Séances de l'Académie des Inscriptions et Belles-Lettres 80/3, p. 205-206; MÉcÉRIAN J. (1934), «Une mission archéologique dans l'Antiochène. Rapport sur la deuxième campagne de fouilles 1933 », Comptes rendus des Séances de l'Académie des Inscriptions et Belles-Lettres 78/2, p. 144-149; id. (1948), "Monastère de Saint-Siméon-Stylite-le-Jeune, exposé des fouilles », Comptes rendus des Séances de l'Académie des Inscriptions et Belles-Lettres 92/3, p. 323-328; id. (1951), «Le monastère de Saint Syméon le Stylite $\mathrm{du}$ Mont Admirable », in Actes du VI Congrès international des études byzantines, Paris 27 juillet 2 août 1948, 2 vol. en 1, École des Hautes Études, Paris, vol. II, p. 299-302; id. (1962), « Les inscriptions du Mont Admirable », Mélanges de l'Université Saint-Joseph 38, p. 297-330; id. (1964), « Expédition archéologique dans l'Antiochène occidentale », MUSJ 40, p. 1-144.
} 
eulogies tardo-antiques en terre cuite avec représentations de saints et de motifs du Nouveau Testament (cat. $\left.\mathrm{n}^{\text {os }} 1-4\right)$, trois moules (cat. ${ }^{\text {os }} 6-8$ ) dont deux à motifs chrétiens, une icône médiévale en stéatite (cat. $\left.{ }^{\circ} 9\right)$, un couvercle de reliquaire (cat. $\mathrm{n}^{\circ} 17$ ), quelques petits objets en pierre à destination inconnue (cat. $\mathrm{n}^{\text {os }} 9-17$ ), de petits récipients en céramique en parfait état de conservation (cat. $\mathrm{n}^{\text {os }} 18-23$ ) et des pipes à tabac ottomanes qui forment le groupe le plus nombreux (cat. $\mathrm{n}^{\text {os }} 24-52$ ).

Aucun des objets présentés ci-après ne fut publié ni mentionné par Jean Mécérian². De tous les petits objets de la collection, seuls furent publiés le sceau monastique du Mont Admirable et l'écuelle en pierre avec représentation du stylite ${ }^{3}$, absents du présent catalogue.

Comme la documentation des travaux archéologiques réalisés dans les années trente est perdue, il est impossible d'attribuer avec certitude la provenance des objets présentés dans ce catalogue à tel ou tel site fouillé par Jean Mécérian. Il est d'ailleurs probable que certains de ces objets aient été découverts en dehors de l'Antiochène occidentale. La collection contient en effet «l'écuelle » en pierre, signalée plus haut, qui a été très certainement acquise sur le marché des antiquités ${ }^{4}$. Il en est vraisemblablement de même d'une des eulogies (cat. $\mathrm{n}^{\circ} 4$ ), et il y a de bonnes raisons de croire qu'il s'agit d'un faux.

Certains des objets étudiés (cat. nos $5,7,18-23,49$ ) figurent sur les photographies de Jean Mécérian conservées à la Bibliothèque Orientale de l'Université SaintJoseph à Beyrouth. Toute cette documentation photographique est répartie dans des enveloppes contenant négatifs et clichés, classés selon le sujet (par ex. architecture, mobilier archéologique) ou le lieu (monastère de Saint-Syméon-Stylite-le-Jeune, église de l'Arbre de la Vie). Nous accompagnons la présentation de chacun de ces objets d'une brève information sur d'autres clichés rangés dans la même enveloppe, ce qui pourrait en effet servir à identifier le lieu de découverte de l'objet, si celui-ci provenait du site auquel se rapportent les autres photos. Cependant, compte tenu de l'absence de données sur le processus d'archivage de tous ces documents, ces informations doivent être traitées avec prudence.

Le présent catalogue contient aussi une eulogie en terre cuite (cat. ${ }^{\circ}{ }^{5}$ ) dont l'actuel lieu de conservation est inconnu. L'unique témoignage de son existence est le cliché conservé dans les archives photographiques du père Jean Mécérian.

\footnotetext{
Voir références n. 2.

3 Sceau: MÉcÉrian, « Monastère de Saint-Siméon-Stylite-le-Jeune, exposé des fouilles », p. 324 ; id., «Les inscriptions du Mont Admirable », p. 304, pl. 2.1 (inv. USJ-23). «Écuelle» en pierre: Mouterde R. (1947), «Nouvelles images de stylites », Orientalia Christiana Periodica 13, p. 246, 247, fig. 4 (inv. JM-Eu-0006).

4 Ibid.
} 


\section{Catalogue}

\section{Eulogies}

\section{Eulogie ronde en terre cuite, argile rose ( $\mathrm{pl}$. I 1)} Inv. no JM-Eu-0001

Diam. env. $1,6 \mathrm{~cm}$; ép. $0,6 \mathrm{~cm}$. L'avers porte un décor moulé en relief: scène de l'adoration des mages dans un cadre circulaire. La représentation est très simplifiée. Le relief ne sert qu'à rendre les contours des personnages. À droite, Marie assise sur une chaise, tenant l'enfant Jésus sur ses genoux. Têtes nimbées. À gauche, trois figures humaines debout à droite. Il faut y voir les trois mages. Revers non décoré avec empreinte de doigt laissée pendant le remplissage du moule. L'adoration des mages fait partie des motifs iconographiques très répandus sur les eulogies en terre cuite qui revêtent la forme de petits jetons ${ }^{5}$. Datation d'après les analogies : milieu du $\mathrm{VI}^{\mathrm{e}}$ ou début du VII ${ }^{\mathrm{e}}$ siècle apr. J.-C. ${ }^{6}$

\section{Eulogie légèrement ovale en terre cuite rouge brun (pl. I 2) Inv. ${ }^{\circ} \mathrm{JM}-\mathrm{Eu}-0002$}

Diam. env. 2,6 cm; ép. 1,3 cm. L'avers porte un décor moulé en relief: scène d'entrée triomphale de Jésus à Jérusalem. Au centre, Jésus monté sur le dos d'un ânon, les deux figures orientées à gauche. Jésus, la tête nimbée, porte un vêtement drapé. Il tient de la main gauche un bâton surmonté vraisemblablement d'une croix.

\footnotetext{
5 En témoigne le mieux la collection d'eulogies de provenance inconnue acquise par le British Museum à un brocanteur londonien en 1973. Elle contient 80 pièces dont 18 (groupe iconographique le plus nombreux) portent la scène de l'adoration des mages: CAMBer R. (1979), «A Hoard of Terracotta Amulets from the Holy Land », in Actes du XVe congrès international d'études byzantines, Athènes septembre 1976, Association internationale des études byzantines, Athènes, p. 99-106. Cf. TchALENKo G. (1958), Villages antiques de la Syrie du Nord, le massif du Bélus à l'époque romaine, 3 vol., (Bibliothèque archéologique et historique, 50) Librairie Orientaliste Paul Geuthner, Paris, vol. III, p. 43, fig. 28. Sur les vertus de protection des eulogies avec représentation de l'adoration des mages, $c f$. VIKAN G. (1991), «Guided by Land and Sea. Pilgrim Art and Pilgrim Travel in Early Byzantium », in Dassmann E. et Thraede K. (éds.), Tesserae. Festschrift für Josef Engemann, (Jahrbuch für Antike und Christentum, Ergänzungsband, 18) Aschendorffsche Verlagsbuchhandlung, Münster, p. 80-82.

6 Fansa M. et Bollmann B. (éds.) (2008), Die Kunst der frühen Christen in Syrien. Zeichen, Bilder und Symbole vom 4. bis 7. Jahrhundert. Begleitband zur Sonderausstellung im Landesmuseum Natur und Mensch Oldenburg, (Schriftenreihe des Landesmuseums Natur und Mensch, 60) Verlag Philipp von Zabern, Mainz am Rhein, cat. no 127; Rahmani L.Y. (1979), « The Adoration of the Magi on Two SixthCentury C.E. Eulogia Tokens », Israel Exploration Journal 29/1, p. 34-36; id. (1993), «Eulogia Tokens from Byzantine Bet She 'an », 'Atiqot 22, p. 112-115; cf. VIKAN, « Guided by Land and Sea », pl. 9, fig. C. L'objet le plus proche du point de vue iconographique est l'eulogie du British Museum: CAMBER, «A Hoard of Terracotta Amulets », p. 101, n 11, fig. 4 (au milieu). Sur la datation, ibid. p. 106.
} 
Au-dessus de la tête de l'animal figure une croix à branches égales. À gauche, une figure masculine debout tenant un rameau de palmier. Le travail de l'objet étant peu soigné, cette figure n'est que partiellement visible. Entre l'ânon et l'homme au rameau se trouve un enfant, les bras tendus vers Jésus. Revers non décoré avec trois empreintes digitales. Certaines eulogies en forme de petits jetons en terre cuite, connues par des publications, sont ornées de scènes représentant le dimanche des rameaux, mais aucun de ces objets ne correspond exactement à celui de la collection Mécérian ${ }^{7}$. Par ailleurs, le diamètre de la pièce examinée est nettement supérieur à celui des eulogies publiées, ce qui laisse ouverte la question de la datation de celle-ci.

\section{Ampoule de Saint Ménas en terre cuite, aplatie, argile brun pâle (pl. I 3)} Inv. no JM-Eu-0004

Diam. de l'ampoule env. $6 \mathrm{~cm}$; ép. max. env. 1,8 cm. La fiole est endommagée à plusieurs endroits. Anses et col brisés. Les deux parois de l'ampoule se sont décollées à la jonction, l'une d'entre elles est cassée en deux. La décoration des deux faces est identique: en bordure, deux cercles concentriques entre lesquels se trouve un filet de perles. Au centre, saint Ménas, debout de face, les bras étendus. Au-dessus de ses bras figurent deux croix à branches égales. Celle de droite est légèrement penchée. Au-dessous des bras du saint, des lignes verticales isolées dessinent le contour de son manteau. La figure centrale est flanquée de deux chameaux disposés symétriquement. Très simplifiée, la décoration reproduit uniquement les contours de ses composants. Datation d'après les trouvailles similaires provenant des fouilles alexandrines : période entre 610 et 650 apr. J.-C. ${ }^{8}$

\footnotetext{
Cf. CAmber, «A Hoard of Terracotta Amulets », p. 104, ${ }^{\text {os }} 45-48$, fig. 9; Dauterman Maguire E., Maguire H. P. et Duncan-Flowers M. J. (1989), Art and Holy Powers in the Early Christian House, (Illinois Byzantine Studies, 2) University of Illinois Press, Urbana, p. 208, nº 129; DalToN O. M. (1901), Catalogue of Early Christian Antiquities and Objects from the Christian East in the Department of British and Mediaeval Antiquities and Ethnography of the British Museum, British Museum, Londres, p. 171, $\mathrm{n}^{\text {os }} 966,967$.

8 Les analogies les plus proches sont les $\mathrm{n}^{\text {os }}$ 56, 62-64 mis au jour à Kôm El-Dikka: KIss Z. (1989), Les ampoules de Saint Ménas découvertes à Kôm el-Dikka (1961-1981), (Alexandrie, 5) Państwowe Wydawnictwo Naukowe, Varsovie; ainsi que les n ${ }^{\text {os }}$ 10-12 du musée du Louvre: MetzGer C. (1981), Les ampoules à eulogie du Musée du Louvre, (Notes et documents des musées de France, 3) Éditions de la Réunion des musées nationaux, Paris. Pour la datation, voir: Kiss, Les ampoules de Saint Ménas, p. 18. La liste la plus complète des ampoules de saint Ménas publiées se trouve dans: LAMBERT Ch. et PEDEMONTE Demeglio P. (1994), « Ampolle devozionali ed itinerary di pellegrinaggio tra IV e VII secolo », Antiquité tardive 2, p. 205-231; GILli M. (2002), Le ampolle di San Mena. Religiosità, cultura materiale e sistema produttivo, (Tardoantico e Medioevo - Studi e strumenti di archeologia, 5) Palombi, Rome.
} 

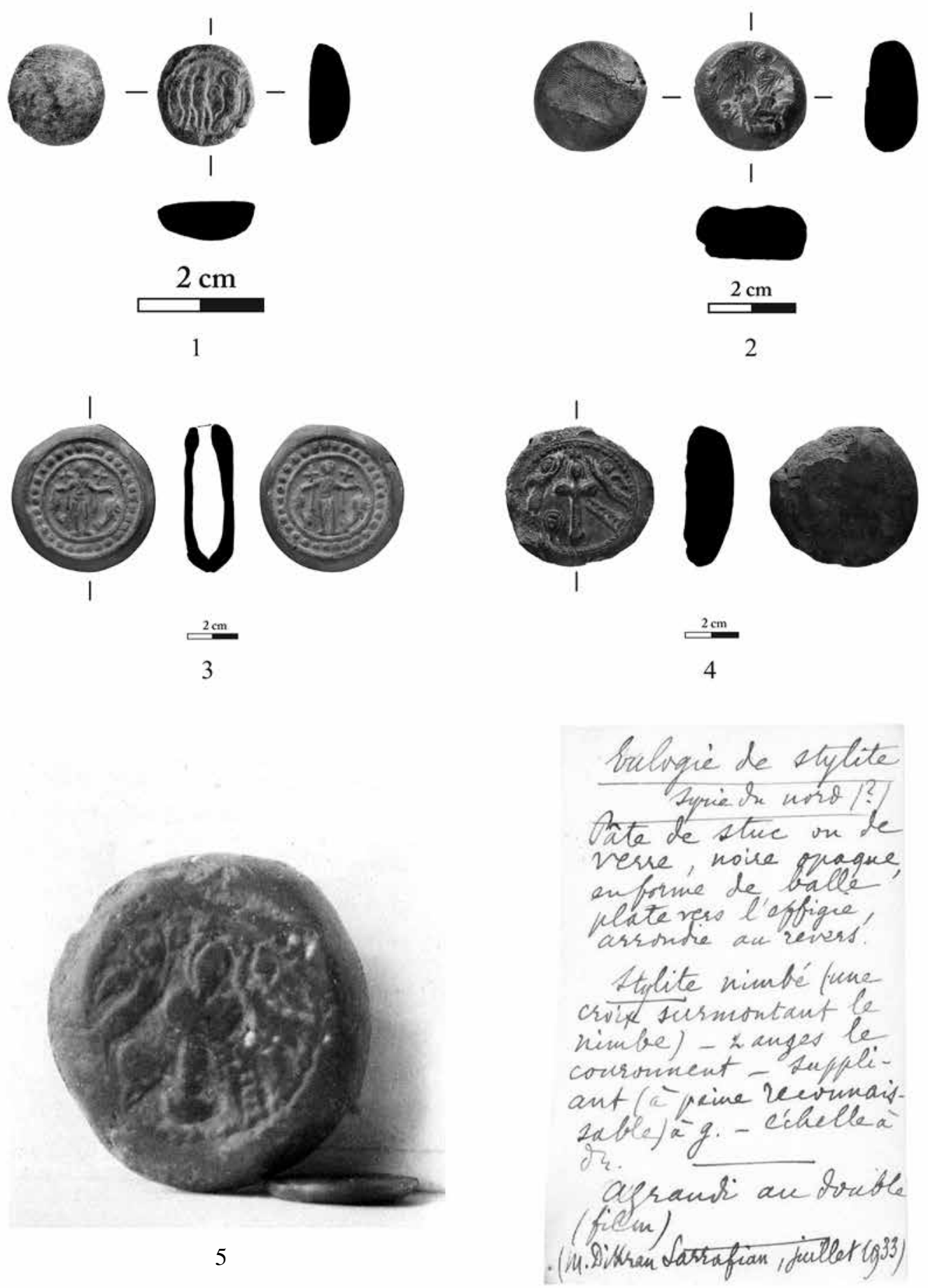

Pl. 1 - Eulogies en terre cuite et ampoules. 
4. Eulogie quasi ronde en pâte céramique rose (pl. I 4) Inv. ${ }^{\circ}$ JM-Eu-0005

Diam. env. 5,3 cm; ép. max. 1,8 cm. L'objet est ébréché sur la plus grande partie de son pourtour. Une seule face est décorée. La décoration se compose d'une scène inscrite dans deux cercles concentriques en bordure. Au centre, un stylite représenté jusqu'à la taille, les bras étendus. Ses membres inférieurs font corps avec la colonne, de sorte que l'ensemble apparaît comme une croix latine avec les extrémités des branches arrondies. Au-dessus de la tête du stylite (c'est-à-dire au-dessus de l'extrémité supérieure de la croix) se trouve une petite croix à branches égales vers laquelle tendent les bras les deux anges volant de part et d'autre de la figure centrale $^{9}$. Au-dessous des anges se trouvent, à droite, une échelle appuyée sur la colonne, et à gauche, un encensoir. Les analogies stylistiques et iconographiques les plus proches de cette scène sont les représentations figurant sur une lampe à huile de Simalî (Syrie), sur deux eulogies du même type et sur des écuelles en céramique ou en pierre publiées antérieurement ${ }^{10}$. L'objet qui mérite une attention particulière est la lampe à huile. Son disque est décoré d'une scène identique ou très proche de celle de l'eulogie de la collection Mécérian, peut-être réalisée au moyen du même moule. Compte tenu du fait que la forme de la petite lampe est très proche de celle des lampes du $\mathrm{III}^{\mathrm{e}}$ et $\mathrm{IV}^{\mathrm{e}}$ siècles apr. J.-C., soit de la période qui précède l'apparition du culte des stylites et le premier stylite, il est tout à fait légitime de croire qu'il s'agit d'un faux. Aussi faut-il considérer l'eulogie en question, ainsi que tous les objets mentionnés dont la décoration présente les mêmes caractéristiques stylistiques, comme des objets contemporains. Un autre argument vient appuyer cette hypothèse, à savoir qu'aucun de ces objets ne provient de la fouille.

\footnotetext{
${ }^{9}$ Sur l'origine probable de ce type de représentation, voir LAFontaine-Dosogne J. (1967), Itinéraires archéologiques dans la région d'Antioche: Recherches sur le monastère et sur l'iconographie de S. Syméon Stylite le Jeune (Bibliothèque de Byzantion, 4) Édition de Byzantion, Bruxelles, p. 149.

${ }^{10}$ Mouterde, « Nouvelles images de stylites », p. 247, 248, fig. 2 (eulogie), fig. 4 (écuelle en pierre); Merlat P. (1949), «Nouvelles images de Saint Syméon le Jeune », in Mélanges d'archéologie et d'histoire offerts à Charles Picard à l'occasion de son $65^{e}$ anniversaire, 2 vol. (= Revue archéologique, 6. sér., t. 29-32), PUF, Paris, vol. II, p. 729, fig. 1.12 (eulogie), p. 729 (lampe à huile); LAFONTAINEDosogne, Itinéraires archéologiques dans la région d'Antioche, p. 149, 150, fig. 82 (eulogie), p. 150, fig. 83 (lampe à huile); Volbach W.F. (1966), «Zur Ikonographie des Styliten Symeon des Jüngeren », in Schumacher W.N. (éd.), Tortulae: Studien zu altchristlichen und byzantinischen Monumenten, (Römische Quartalschrift für christliche Altertumskunde und Kirchengeschichte, Supplementheft, 30) Herder, Rome/Freiburg/Vienne, p. 294, pl. 74, fig. A (écuelle en céramique). L'écuelle en pierre publiée par René Mouterde fait aujourd'hui partie de la collection du père Jean Mécérian (inv. JM-Eu-0006).
} 


\section{Eulogie en terre cuite en forme de jeton circulaire (pl. I 5)}

Sans $n^{\circ}$ d'inv.

La photo de cette eulogie est classée dans l'enveloppe $n^{\circ} 7$ du PCMA à côté de celle de 1'eulogie publiée par René Mouterde ${ }^{11}$. L'objet vient de la collection Dikran Sarrafian. Au dos de la photo figure la notice suivante: «Eulogie de stylite. Syrie du nord (?) Pâte de stuc ou de verre, noire opaque, en forme de balle plate vers l'effigie arrondie au revers. Stylite nimbé (une croix surmontant le nimbe) - 2 anges le couronnent - suppléant (à peine reconnaissable) à g. - échelle à dr. Agrandi au double (film). (M. Dikran Sarrafian, juillet 1933)». L'avers de l'eulogie porte une représentation de stylite formant corps avec la colonne. Sa tête est surmontée d'une petite croix. De part et d'autre de la figure centrale, deux anges tenant chacun une couronne dans les mains. Au bas de la scène se trouvent une échelle (à droite) et un objet arrondi difficilement reconnaissable (à gauche). Il s'agit sans doute d'un encensoir ou d'un vase, comme sur d'autres représentations de ce type datées de la fin du vi /début du VII ${ }^{\mathrm{e}}$ siècle apr. J.-C. ${ }^{12}$ Une des eulogies publiées par Jean Lassus en est une analogie parfaite ${ }^{13}$. Les deux objets sont très vraisemblablement issus du même moule.

\footnotetext{
${ }^{11}$ Mouterde, « Nouvelles images de stylites », p. 247, fig. 2, 3.

12 Pieri D. (2009), «Saint-Syméon-le-stylite (Syrie du Nord): Les bâtiments d'accueil et les boutiques à l'entrée du sanctuaire », Comptes rendus des Séances de l'Académie des Inscriptions et Belles-Lettres 153/4, p. 1414, fig. 19.

${ }^{13}$ Lassus J. (1932), «Images de stylites », Bulletin d'études orientales 2, p. 73, no 4, pl. 19. Quelques autres eulogies de stylite à l'iconographie semblable: Merlat, « Nouvelles images de Saint Syméon le Jeune », cat. 6-10; ElbERN V. H. (1965), «Eine frühbyzantinische Reliefdarstellung des älteren Symeon Stylites », Jahrbuch des Deutschen Archäologischen Instituts 80, p. 280-304, fig. 6, 7 ; FERNANDEZ R. (1975), «Le culte et l'iconographie des stylites », in Peña I., Castellana P. et Fernandez, R. (éds.), Les Stylites syriens, (Studium Biblicum Franciscanum, Collection minor, 16) Franciscan Printing Press, Milan, p. 163-217, fig. 27, 28, 29; LAFONTAINE-Dosogne, Itinéraires archéologiques dans la région d'Antioche, fig. 84-93; id. (1981), «Une eulogie inédite de St Syméon Stylite le Jeune », Byzantion 51/2, p. 631-634; FERnANDEz R. (1985), «Les représentations des stylites », Studia Orientalia Christiana, Collectanea 18, p. 115-151, fig. 11, 13, 14, 15; pl. 2.6, 2.8; DJoBADZE W. (1986), Archaeological Investigations in the Region West of Antioch on-the-Orontes, (Forschungen zur Kunstgeschichte und christlichen Archäologie, 13) Franz Steiner Verlag, Wiesbaden, pl. 57, fig. 221-223. 225; SodinI J.-P. (1989), « Remarques sur l'iconographie de Syméon l'Alépin, le premier stylite », Monuments et Mémoires. Fondation Eugène Piot 70, p. 29-53, fig. 4, 6, 9, 11-13, 16; id. (1993), « Nouvelles eulogies de Syméon », in Jolivet-LÉvy C., KAPLAN M. et Sodini J.-P. (éds.), Les saints et leur sanctuaire à Byzance: textes, images et monuments (Byzantina Sorbonensia, 11) Publication de la Sorbonne, Paris, fig. 2-18; Fansa et Bollmann, Die Kunst der frühen Christen in Syrien, cat. 131.
} 


\section{Objets en pierre}

\section{Fragment de moule à flans en basalte noir (pl. II 6)}

Sans $n^{\circ} d^{\prime}$ inv.

Long. et larg. de la partie préservée, respectivement $9,8 \mathrm{~cm}$ et $8,9 \mathrm{~cm}$; ép. max. $1,6 \mathrm{~cm}$. Dans un des coins se trouvent trois alvéoles circulaires à fond tronconique. Une seule alvéole est préservée intacte (diam. 3,5 cm). Les alvéoles sont reliées les unes aux autres par deux types de petites rigoles: rigoles étroites et assez profondes et rigoles plus larges et peu profondes. Disposées perpendiculairement, les rigoles servaient à alimenter les cavités de métal en fusion; les rigoles plus étroites pouvaient en plus servir à évacuer l'air. Les négatifs de ces rigoles sont parfois visibles sur les pourtours de monnaies frappées et de sceaux de plomb. Dans l'unique coin intact du moule se trouve une petite quantité de plomb informe qui semble boucher l'orifice ayant servi à bien ajuster l'autre valve du moule. Vu la très faible profondeur des alvéoles - à peine $0,08 \mathrm{~cm}$ (sans compter les étroites rigoles $-0,1 \mathrm{~cm}$ ), il y a lieu de penser que l'autre valve était munie d'un jeu de cavités identique ou semblable. Compte tenu des analogies connues, il faudrait voir dans cet objet un moule à flans ayant servi à fabriquer des sceaux de plomb très répandus à l'époque byzantine ${ }^{14}$.

\section{Fragment de moule en basalte noir (pl. II 7)}

Inv. $\mathrm{n}^{\circ}$ USJ-27

Trois côtés partiellement conservés, avec ébréchures dans les coins et sur le dessous. Long. du fragment préservé: 11,6 cm. En admettant que l'objet moulé fût rond, la longueur minimale du moule serait de $14,8 \mathrm{~cm}$. Largeur et épaisseur respectivement $12,8 \mathrm{~cm}$ et $2,5 \mathrm{~cm}$. La décoration se compose de deux cercles de $11,2 \mathrm{~cm}$ de diamètre en bordure, d'une croix latine dont les branches sont terminées par de petits cercles et de rinceaux de vigne qui partent d'un vase ${ }^{15}$. Le relief est si peu profond qu'il est difficile de le faire reproduire sur le dessin en coupe du moule. Le côté court du moule présente un petit canal en entonnoir qui servait à alimenter le moule de métal en fusion. Dans le coin le mieux préservé, sur le dessous, se trouve une ouverture avec un clou en fer dont il ne subsiste qu'un menu fragment. Le clou servait sans doute à fermer les deux parties du moule. La nature de l'objet fabriqué

\footnotetext{
${ }^{14}$ Weinberg Davidson G.R. (1952), The Minor Objects, (Corinth, 12) The American School of Classical Studies at Athens, Princeton, p. 311, 328, 329, n 2828-2831.

${ }^{15}$ Le motif de rinceau partant d'un vase fait partie des motifs décoratifs les plus répandus dans les mosaïques, les peintures et les bas-reliefs romains et ceux du début de la période byzantine dans tout le bassin de la Méditerranée.
} 

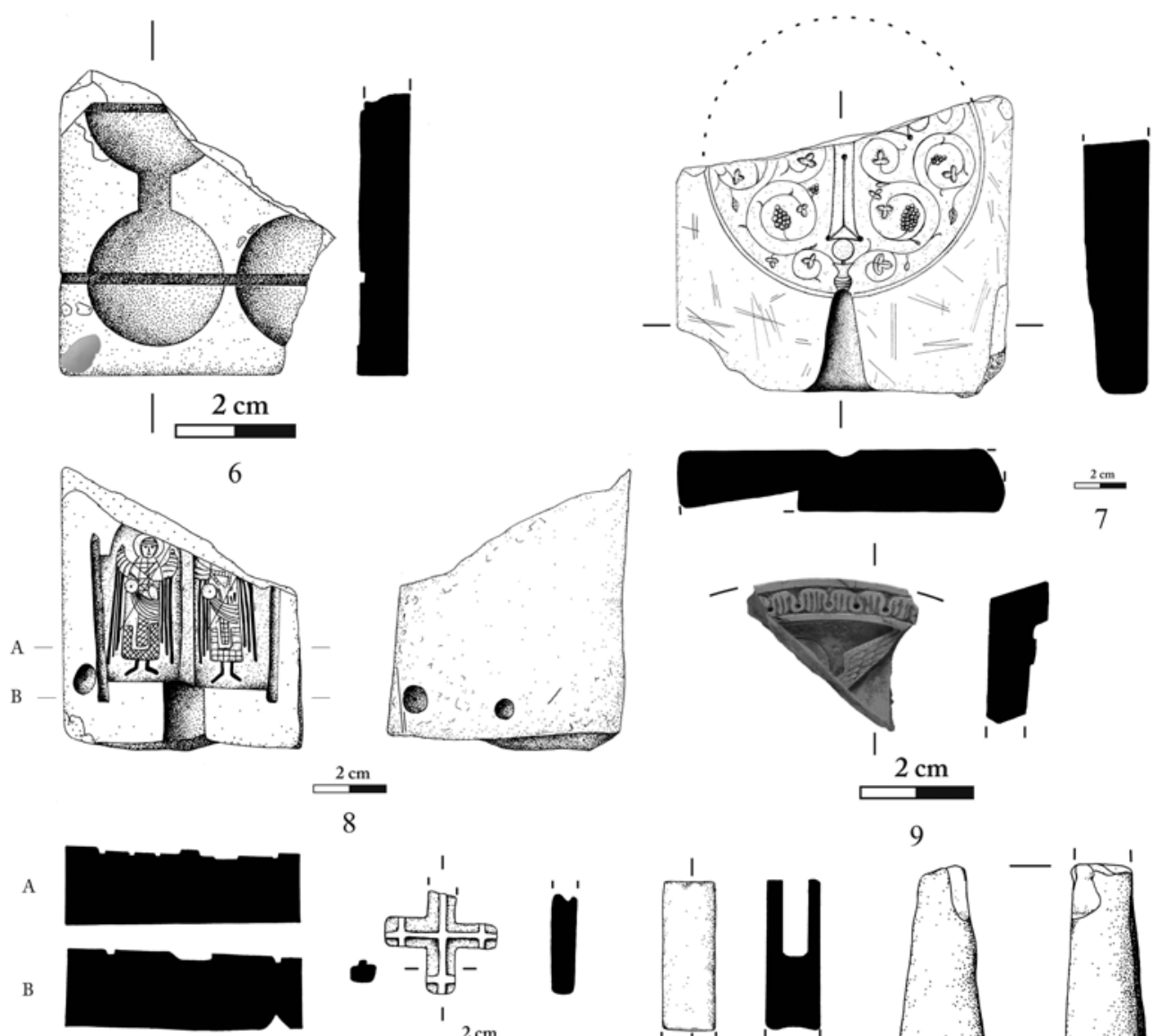

$\frac{2 \mathrm{~cm}}{8}$

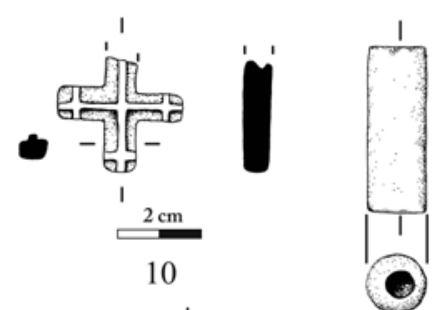

9

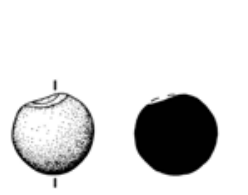

(-)

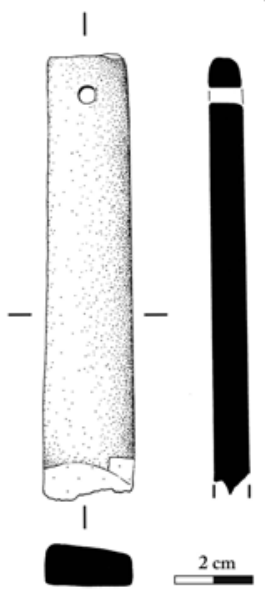

15
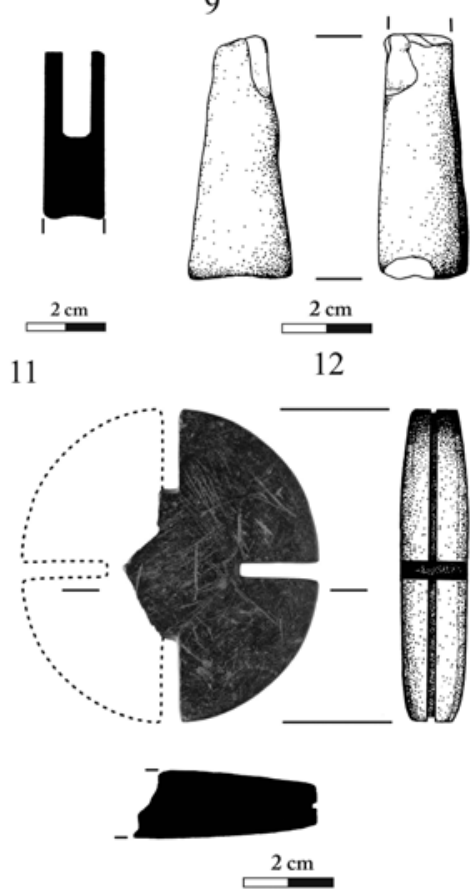

16

Pl. II - Objets en pierre. 
dans ce moule pose problème. Vu les dimensions, la forme et les motifs décoratifs d'inspiration chrétienne, il faudrait envisager comme analogie le miroir byzantin en bronze avec représentation d'un saint, conservé à la Barakat Gallery ${ }^{16}$. Mais, il est possible d'envisager aussi une plaquette décorative ou un type peu répandu de croix de procession, ou encore un rhipidion. Un argument va toutefois à l'encontre de la première de ces hypothèses, à savoir que la petite ouverture, par laquelle était versé le métal, chevauche en partie la bordure et la représentation du vase d'où partent les rinceaux de vigne. D'autres arguments contredisent la deuxième hypothèse. Tout d'abord, la plupart des croix de procession étaient fixées à la hampe au moyen d'une tige placée à l'extrémité inférieure de la croix. Ensuite, le diamètre de la plaque est relativement petit par rapport à celui de certaines croix de procession tardo-antiques connues ${ }^{17}$. Enfin, les dimensions de l'objet métallique ne correspondent pas à celles du rhipidion dont le diamètre, aussi bien dans l'Antiquité que de nos jours, dépasse en règle générale $20 \mathrm{~cm}^{18}$. Compte tenu de toutes ces réserves, aucune des hypothèses émises ne peut être définitivement rejetée. Les clichés de cet objet se trouvent dans les enveloppes contenant la documentation photographique de Jean Mécérian ${ }^{19}$.

\section{Fragment de moule en basalte noir (pl. II 8)}

Inv. $\mathrm{n}^{\circ}$ USJ-25

Larg. 6,6 cm; ép. 2,2 cm; long. du fragment préservé max. 7,5 cm. Le haut du moule est brisé. Au bas, reste visible une large rigole à section trapézoïdale qui servait à alimenter le moule de métal en fusion. Sur le dessus décoré ainsi que sur le dessous

${ }^{16}$ AdresseWeb : http://www.barakatgallery.com/store/index.cfm/FuseAction/ItemDetails/UserID/0/CFID/ 16663023/CFTOKEN/a1ee8d062ff885d5-06168599-3048-33BC-FCDE4CDCA44DE780/jsessionid/84 30a3fde6aedb17ed7a1b9143f536022313/CategoryID/65/SubCategoryID/963/ItemID/25733.htm18:37, 22.02.2012.

${ }^{17}$ Cf. Cotsonis J.A. (1994), Byzantine Figural Processional Crosses : Catalogue of an Exhibition at Dumbarton Oaks, 23 September 1994 - 29 January 1995, (Dumbarton Oaks Byzantine Collection Publications, 10) Dumbarton Oaks Research Library and Collections, Washington (D.C.), passim. BagatTi B. (1972), « Il cristianesimo ad Eleuteropoli (Beir Gebrin) », Liber Annuus 22, p. 115, fig. 1, donne un exemple de croix de procession avec douille à la place de la tige de fixation.

${ }_{18}$ Mango M. M. (1986), Silver from Early Byzantium. The Kaper Koraon and Related Treasures, Walters Art Gallery, Baltimore, p. 147, 150-151; van Lohuizen-Mulder M. (1988), « The Cathedra of St. Mark in Venice », Bulletin Antieke Beschaving 63, p. 171; UlBerT Th. (1990), Der kreuzfahrerzeitliche Silberschatz aus Resafa-Sergiupolis, (Resafa, 3) Philipp von Zabern, Mainz am Rhein, pl. 55b. Pour plus d'informations sur le rhipidion, voir ButLer A. J. (1884), The Ancient Coptic Churches of Egypt, 2 vol., Clarendon Press, Oxford, p. 46-53.

${ }^{19}$ Il s'agit des enveloppes aux numéros et aux contenus suivants : 3 PCMA : surtout architecture du Mont Admirable, mais aussi reliefs provenant du même site ainsi que d'autres sites fouillés ; 26 PCMA - plans de Qal'at Sem‘an, reliefs présentés par Dagmara Wielgosz dans ce volume, décorations architecturales du Mont Admirable, objets décorés en pierre de provenance inconnue; 43 PCMA : décorations architecturales et reliefs du Mont Admirable et d'un site qui m'est inconnu. 
non décoré se trouvent des trous coniques ayant servi à assembler et ajuster les deux parties du moule. Au centre, figurent deux anges debout de face. Ces figures sont inscrites dans deux champs rectangulaires travaillés en relief plus plat. À l'origine, le champ de gauche se terminait vraisemblablement en quart de cercle. La partie droite de la décoration devait faire pendant à la gauche. Les deux anges sont représentés de la même façon. Vêtus tous deux d'une chlamyde et d'un loros, ils tiennent à la main gauche (droite au positif) une lance et à la main droite (gauche au positif) un disque rond. La tête de l'ange de gauche est nimbée. La figure de droite est préservée jusqu'à la hauteur des épaules. La présence de lances et de disques permet de voir dans ces deux personnages les archanges Michel et Gabriel (princeps aetherius) ${ }^{20}$. Les deux figures sont séparées par une barre verticale dont la hauteur est égale à celle de la partie non décorée du moule. Sur les bords opposés du champ décoré se trouvent des rainures verticales en relief profond. Après le moulage, une fois la masselotte coupée, les parties gauche et droite de la décoration formaient deux battants de porte indépendants tournant sur des pivots verticaux. Cette petite porte pouvait servir de fermeture de reliquaire ou de triptyque, pour citer en exemple le triptyque de Hahuli en Géorgie, daté de la première moitié du XII siècle apr. J.-C. ${ }^{21}$

\section{Fragment d'icône en stéatite vert pâle (pl. II 9)}

Sans $n^{\circ}$ d'inv.

Ép. max. du fragment préservé $1 \mathrm{~cm}$. Le dos poli mais non décoré. Sur l'avers une aile et un fragment de nimbe. Les analogies connues permettent de supposer que l'icône était rectangulaire, terminée en haut par un demi-cercle. En témoigne l'unique bord préservé qui revêt la forme d'un cadre arrondi décoré de palmettes. Cette décoration faisait sans doute partie d'un arc surmontant deux petites colonnettes. Dans ce type d'icônes, le rapport largeur/hauteur est de 2 à 3 . Le fragment de demi-cercle préservé permet d'estimer la largeur de l'objet à $9 \mathrm{~cm}$. Sa hauteur serait donc d'env. $13 \mathrm{~cm}$, ce qui permet de classer cet objet dans la catégorie d'icônes en stéatite de taille moyenne ${ }^{22}$. La décoration est réalisée en relief peu profond, ce qui est caractéristique des $\mathrm{XI}^{\mathrm{e}}$ et XII ${ }^{\mathrm{e}}$ siècles. De même, la présence d'arceau décoré de palmettes est typique

${ }^{20}$ Cf. Jolivet-Lévy C. (1998), « Note sur la représentation des archanges en costume impérial dans l'iconographie byzantine », Cahiers Archéologiques 46, p. 121-128.

21 Alpago-Novello A., Beridze V. et Lafontaine-Dosogne J. (1980), Art and Architecture in Medieval Georgia, (Publications d'histoire de l'art et d'archéologie de l'Université Catholique de Louvain, 21) Inst. Sup. d'Archéologie et d'Histoire de 1'art, Louvain-La-Neuve, p. 70, fig. 70. Exemples de reliquaires fermés par de petites portes: Grabar A. (1950), «Quelques reliquaires de saint Démétrios et le martyrium du saint à Salonique », Dumbarton Oaks Papers 5, p. 3-28.

${ }^{22}$ Kalavrezou-Maxeiner I. (1985), Byzantine Icons in Steatite, 2 vol., (Byzantina Vindobonensia, 15) Verlag der Österreichischen Akademie der Wissenschaften, Vienne, vol. I, p. 27. 
de cette période ${ }^{23}$. Cependant, compte tenu de l'état fragmentaire de l'objet, cette datation reste purement hypothétique.

10. Croix en pierre (pl. II 10)

Inv. n JM-Eu-0003

Larg. $3 \mathrm{~cm}$; long. du fragment préservé $2,8 \mathrm{~cm}$; ép. max. $0,7 \mathrm{~cm}$. Une des branches de la croix est cassée à son extrémité. À l'avers, traits convexes croisés formant l'angle droit. Leurs extrémités sont terminées par de petites croix. La partie cassée de la branche devait sans doute porter le même décor. Vu que le contour de la branche cassée s'évase comme celui des trois branches intactes, il y a lieu de croire qu'il s'agissait d'une croix à branches égales. Datation indéterminée.

\section{Objet cylindrique en marbre blanc (pl. II 11)}

Sans $n^{\circ}$ d'inv.

Long. du fragment préservé $4,1 \mathrm{~cm}$; diam. de l'objet $1,5 \mathrm{~cm}$; diam. de l'orifice $0,6 \mathrm{~cm}$. Surface polie, un orifice circulaire profond à l'une des extrémités. L'autre extrémité brisée. Datation indéterminée.

12. Objet à fonction non identifiée (pl. II 12)

Sans no d'inv.

Long. du fragment préservé $5 \mathrm{~cm}$; larg. 1,8 cm. Prisme tronqué en pierre brun clair à surface polie. Extrémité plus étroite cassée. Extrémité plus large en queue d'hirondelle. Section rectangulaire. Datation indéterminée.

\section{Boule en calcaire (pl. II 13)}

Sans $n^{\circ} d^{\prime}$ inv.

$2,5 \mathrm{~cm}$ de diamètre. Petite ébréchure de surface. La fonction de l'objet prête à discussion; il s'agit soit d'un pion de jeu de type mancala ou d'un instrument de chasse: boule utilisée dans la chasse aux oiseaux (genre de boléador) ${ }^{24}$. Datation indéterminée.

\footnotetext{
${ }^{23}$ Ibid., p. 33, 53, 57.

${ }^{24}$ Hillbom N. (2000-2001), « Minoan and Eastern Mediterranean Games and Game Boards: A History of Research », Opuscula Atheniensia 25-26, p. 53-65. Sur le jeu même, voir De Voogt A. (2010), « Mancala Players at Palmyra », Antiquity 84/326, p. 1055-1066.
} 
14. Pierre gris bleu (pl. II 14)

Sans $n^{\circ}$ d'inv.

Haut. du fragment préservé $2,8 \mathrm{~cm}$; diam. 2,9 cm; diam. de l'orifice $1 \mathrm{~cm}$. À section circulaire avec un trou conique sur l'une des extrémités. L'autre extrémité brisée. Sur un côté et autour du trou conique traces de tournage. Datation indéterminée.

\section{Fragment de pierre à aiguiser en grès gris (pl. II 15)}

Sans $n^{\circ}$ d'inv.

Larg. max. 2,3 cm; ép. $1 \mathrm{~cm}$; long. du fragment préservé $11,7 \mathrm{~cm}$. L'extrémité plus large brisée. À l'autre extrémité un trou permettant de suspendre la pierre. L'outil porte des traces d'usage sous forme de rayures blanches. Sans connaître le contexte de sa découverte, il est impossible de proposer une datation, même approximative. Les pierres à aiguiser de ce type sont connues depuis l'âge du bronze jusqu'à l'époque contemporaine.

\section{Fragment de disque circulaire (pl. II 16)}

Sans $n^{\circ}$ d'inv.

Ép. $1,5 \mathrm{~cm}$; diam. reconstitué $6,9 \mathrm{~cm}$. En basalte noir de très bonne qualité, avec trois encoches disposées à distance régulière. Petite rainure sur le pourtour. Toutes les surfaces polies. Datation indéterminée.

\section{Couvercle de reliquaire en marbre en forme de toit à deux pentes (pl. III 17)} Inv. ${ }^{\circ}$ JM-S-4006, USJ-20

23,3 x 15,6 x 8,8 cm. Les coins portent des traces de quatre acrotères brisés. Au milieu d'une des pentes se trouve un orifice par lequel était versée l'huile dans la caisse à reliques ou par lequel elle était recueillie ${ }^{25}$. Le couvercle présente une forme

\footnotetext{
${ }^{25}$ Dans l'église est de Pella et dans l'église nord-ouest d'Hippos, au moment de la découverte des reliquaires, les trous étaient munis respectivement d'un petit tuyau et d'une baguette. Ces dispositifs permettaient sans doute aux croyants de communiquer indirectement avec les reliques. McNicoll A.W. et al. (1992), Pella in Jordan 2. The Second Interim Report of the Joint University of Sydney and College of Wooster Excavations at Pella, 1982-1985, (Mediterranean Archaeology Supplement, 2) Meditarch, Sydney, p. 158, 159, pl. 106, 107a; Segal A. et al. (2003), Hippos-Sussita. Fourth Season of Excavations, June - July 2003, Zinman Institute of Archaeology, University of Haifa, Haifa, p. 26, fig. 48. Sur le culte des reliques et des reliquaires, voir: CAnivet M.-T. (1978), «Le reliquaire à huile de la grande église de Huarte (Syrie) », Syria 55/1-2, p. 153-162; GESSEL W. (1988), « Das Öl der Märtyrer. Zur Funktion und Interpretation der Ölsarkophage von Apamea in Syrien », Oriens Christianus 72, p. 183-202; DonCEel-VoûTe P. (1995), « Le rôle des reliquaires dans les pèlerinages ", in Dassman E. et Engemann J. (éds.), Akten des XII. Internationalen Kongresses für christliche Archäologie, Bonn 22.-28. September 1991, 2 vol., (Jahrbuch für Antike und
} 
bien originale: un des ses côtés courts semble indiquer que le couvercle pouvait être posé et en même temps glissé sur la caisse à reliques ${ }^{26}$. La forme du couvercle à acrotères est typique des reliquaires tardo-antiques.

\section{Petits récipients en céramique}

\section{Unguentarium fusiforme (pl. III 18)}

Inv. $n^{\circ}$ JM-C-3002

Haut. $11,3 \mathrm{~cm}$; vol. $39 \mathrm{~cm}^{3} .{ }^{27}$ Très bon état de conservation, avec petite ébréchure de surface. Bord à lèvre repliée sur l'extérieur; col évasé vers le haut; panse bombée. Pied cylindrique de taille moyenne, évasé vers le haut et vers le bas. Base plate. La surface de la fiole est polie et recouverte d'engobe jaune rougeâtre (5 YR 7/6) partiellement effacé. Couleur de l'argile à la cassure: extérieur brun (7.5 R 4 juin); intérieur jaune rougeâtre (5 YR 5/8). Le récipient est proche par sa forme du type IV pisidien hellénistique, selon la typologie d'Ergün Lafli ${ }^{28}$.

\section{Unguentarium fusiforme (pl. III 19)}

Inv. $n^{\circ}$ JM-C-3003

Base plate. Haut. $15,5 \mathrm{~cm}$; vol. $56 \mathrm{~cm}^{3}$. Très bon état de conservation. Bord à lèvre repliée sur l'extérieur. Long col évasé. Panse élancée au bas du récipient, dans la partie supérieure une légère rupture de pente. Absence de rupture de ligne entre la

Christentum Ergänzungsband, 20), Aschendorffsche Verlagsbuchhandlung et Pontificio Istituto di archeologia cristiano, Münster/Cité du Vatican, vol. I, p. 184-205; PEÑA I. (2000), Lieux de pèlerinage en Syrie, (Studium Biblicum Franciscanum, Collectio Minor, 38) Franciscan Printing Press, Milan p. 49-59.

${ }^{26}$ Ce type de fermeture s'observe aussi sur le reliquaire provenant d'Otranto (Italie), D'AngELA C. (1995), « Recenti scoperte paleocristiane ad Otranto », Corso di cultura sull'arte ravennate e bizantina 42, p. 282, fig. 4-9.

${ }^{27}$ Le volume a été calculé au moyen du logiciel AutoCad, avec, comme données de départ, les dessins des coupes des récipients. Cependant, compte tenu de l'excellent état de conservation de ces objets, il a été impossible de mesurer avec précision l'épaisseur des parois. C'est pourquoi les valeurs proposées doivent être considérées comme approximatives. La couleur de l'argile a été déterminée d'après Munsell Soil-color Charts (version 2009).

${ }^{28}$ LAFLI E. (2003a), Studien zu hellenistischen, kaiserzeitlichen und spätantik-frühbyzantinischen Tonunguentarien aus Kilikien und Pisidien (Südtürkei): der Forschungsstand und eine Auswahl von Fundobjekten aus den örtlichen Museen, Universität zu Köln, (thèse de doctorat inédite), p. 85. Cf. forme VI dans $i d$. (2003b), «Hellenistische, römischkaiserzeitliche und spätantik-frühbyzantinische Tongunguentarien aus dem Museum von Gaziantep (Südosttürkei) », Instrumentum 18 (December), p. 32. Cet unguentarium ainsi que les $\mathrm{n}^{\text {os }}$ 19, 20, 21, 22 figurent sur une photo des archives de J. Mécérian (enveloppe $\mathrm{n}^{\circ} 40$ PCMA). Cette enveloppe contient en outre les photos de céramiques glaçurées, de lampes à huile et d'unguentaria en verre faisant partie de la même collection $-c f$. les articles de Tasha Vorderstrasse et Tomasz Waliszewski dans le présent volume. L'enveloppe porte l'inscription « Octogone ». Il s'agit très vraisemblablement de 1'octogone du monastère de Saint-Syméon-Stylite-le-Jeune. 

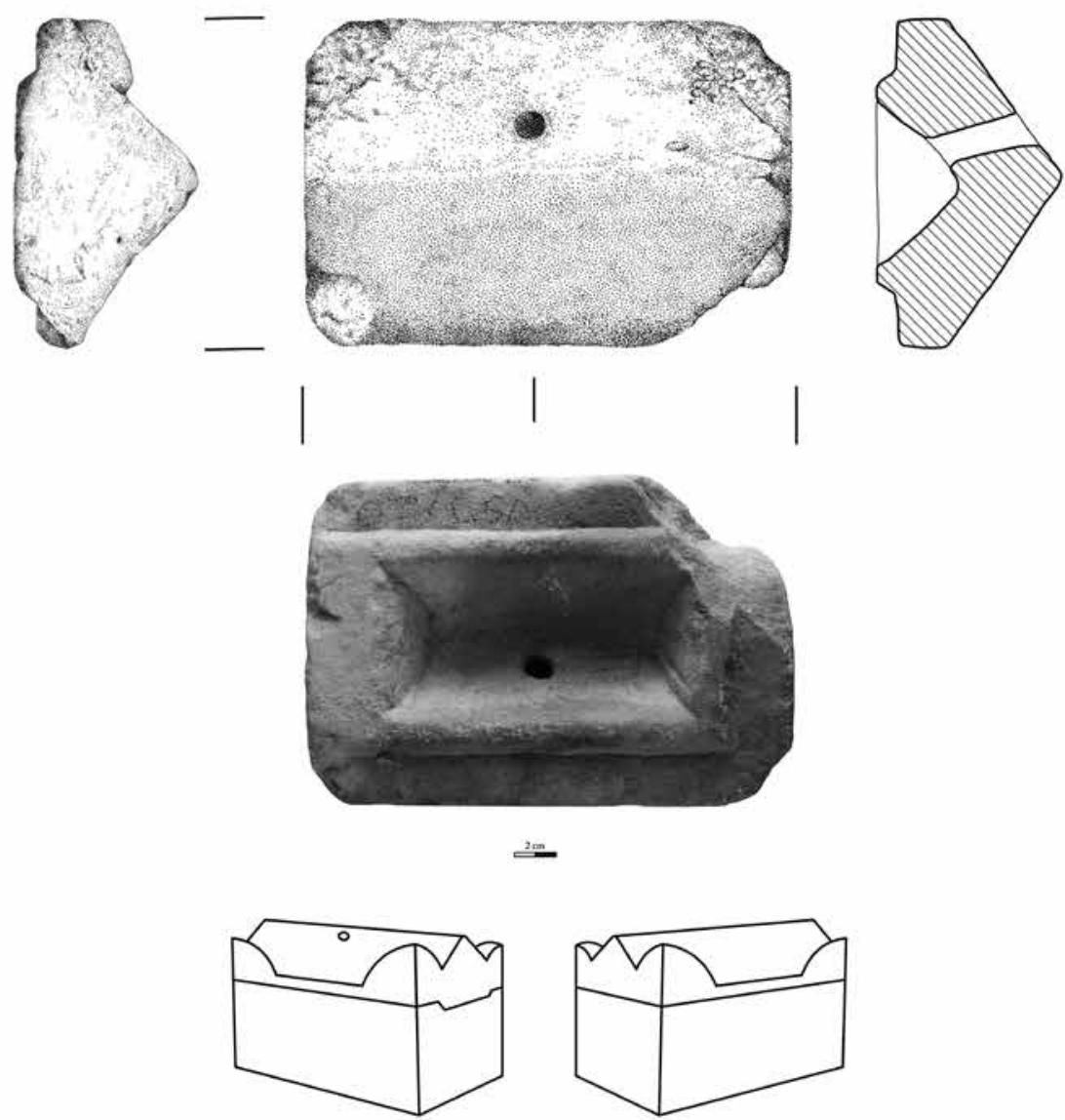

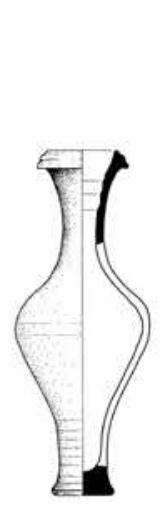

18

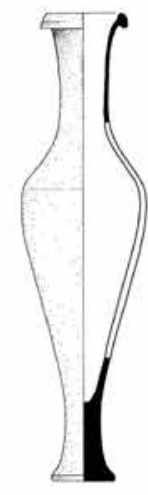

19

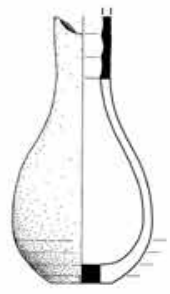

20

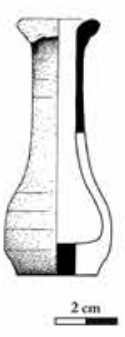

21

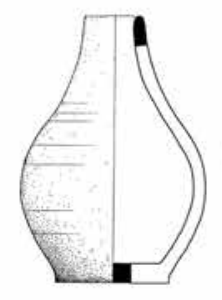

22
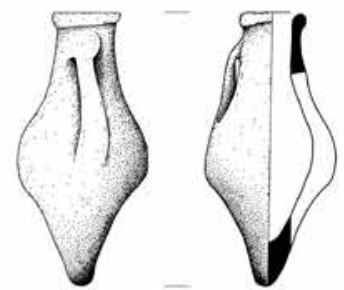

23

Pl. III - Reliquaire en marbre et unguentarii en céramique. 
panse et le pied. Argile rouge (2.5YR 4.6). L'unguentarium (cat. $\left.{ }^{0} 19\right)$ est proche par sa forme du type cilicien XII d'après la typologie d'E. Lafli ${ }^{29}$ attribué à la période hellénistique tardive. Les fioles fusiformes 18 et 19 représentent une des formes les plus répandues d'unguentaria, présente dans toute la région de la Méditerranée à l'époque hellénistique.

20. Unguentarium piriforme à bord cassé (pl. III 20) Inv. no JM-C-3004

Haut. du fragment préservé $8,6 \mathrm{~cm}$; vol. env. $43-56 \mathrm{~cm}^{3}$. Argile rose (5 YR 8/4) faiblement poreuse avec des inclusions blanches. Col légèrement évasé. Base plate. Le récipient est une imitation en céramique des unguentaria en verre du $\mathrm{I}^{\mathrm{er}}$ siècle apr. J.-C. ${ }^{30}$

\section{Unguentarium conservé intact (pl. III 21)} Inv. n JM-C-3001, USJ-C/5

Haut. $8,3 \mathrm{~cm}$; vol. $18 \mathrm{~cm}^{3}$. Bord à lèvre repliée sur l'extérieur; long col cylindrique; panse arrondie; base plate et peu élevée. Argile jaune rougeâtre (7.5 YR 8/6). Datation d'après les analogies, ${ }^{\text {er }}$ siècle apr. J.-C. ${ }^{31}$

22. Bouteille piriforme (pl. III 22)

Inv. n JM-C-3000, USJ-C/1

Haut. $8,6 \mathrm{~cm}$; vol. $84 \mathrm{~cm}^{3}$. Conservée intacte, à bord droit et col court. Panse rebondie, absence de rupture de pente entre le col et la panse. Fond plat avec des traces d'outil qui a permis de décoller la bouteille du tour de potier. Argile rose blanc (2.5 YR 8/2). Point d'analogies. Datation indéterminée.

\section{Bouteille fusiforme (pl. III 23)}

Inv. n JM-C-3005, USJ-C/7

Haut. $9,1 \mathrm{~cm}$; vol. $22 \mathrm{~cm}^{3}$. Lèvre épaissie et repliée sur l'extérieur, légèrement ébréchée. Col court s'amincissant vers le haut; anse unique à section plate reliant le col à la partie supérieure de la panse; pied cônique arrondi à la pointe. Au bas du récipient

\footnotetext{
${ }^{29}$ Lafli, Studien, p. 77, 78. Cf. type V dans: id. « Hellenistische », p. 32.

30 Anderson-Stojanović V. R. (1987), "The Chronology and Function of Ceramic Unguentaria», American Journal of Archaeology 91/1, p. 110. Cf. GöKÇAY M. et AsAL R. (2008), « Excavations in Küçük Ayasofya Street, Eminönü, Istanbul », Anatolia Antiqua 16, p. 222, nº 18, fig. 29.

${ }^{31}$ Anderson-Stojanović, « The Chronology and Function of Ceramic Unguentaria », p. 111-112.
} 
traces d'engobe rouge foncé (2.5 YR 3/6). Argile jaune rougeâtre (5 YR 7/7). La bouteille figure sur une des photos des archives de Jean Mécérian ${ }^{32}$.

\section{Pipes ottomanes ${ }^{33}$}

\section{Fragment de pipe (pl. IV 24)}

Inv. $n^{\circ}$ JM-P-0101

Long. 4,5 cm + ; haut. $1,8 \mathrm{~cm}$; diam. t. $0,5 \mathrm{~cm}^{34}$. Tige terminée en entonnoir et décorée à la roulette. Argile gris très foncé (gley $13 / \mathrm{N}$ ). Faible quantité d'inclusions noires (jusqu'à $0,03 \mathrm{~cm}$ ), mais la majeure partie beaucoup plus petite. Absence de porosité. Vu le petit diamètre de la tige et la couleur de la pâte, il y a lieu de penser qu'il s'agit d'une pipe ancienne (XVI ${ }^{\mathrm{e}}$-XVIII ${ }^{\mathrm{e}}$ siècle).

\section{Fragment de pipe (pl. IV 25)}

Inv. $\mathrm{n}^{\mathrm{o}} \mathrm{JM}-\mathrm{P}-0088$

Long. 3,8 cm +; haut. $2 \mathrm{~cm} \mathrm{+;} \mathrm{diam.} \mathrm{t.} 0,5 \mathrm{~cm}$. Tige partiellement conservée terminée par un anneau conique; bague non décorée. Décor à la roulette sur la tige. Bol cassé. Argile rose blanc (5 YR 8/2). Présence de petites inclusions (jusqu'à $0,03 \mathrm{~cm}$ ). Compte tenu du petit diamètre de la tige, de la couleur de la pâte et de la présence d'embout sous forme d'anneau conique, il faudrait y voir une pipe ancienne ( $\mathrm{XVI}^{\mathrm{e}}$ $\mathrm{XVIII}^{\mathrm{e}}$ siècle).

\footnotetext{
${ }^{32}$ Cette photographie se trouve dans l'enveloppe 14 PCMA, qui contient en outre des photos du monastère de Saint-Syméon-Stylite-le-Jeune du Mont Admirable, des monnaies romaines, une inscription géorgienne et un dessin d'objets en os, très vraisemblablement préhistoriques.

${ }^{33}$ La caractérisation et la datation des pipes que nous proposons dans le présent article s'appuient sur la typochronologie de Rebecca Robinson élaborée à partir du matériel archéologique provenant de Grèce et des dessins de voyageurs: Robinson R. (1983), «Clay Tobacco Pipes from the Kerameikos », Mitteilungen des Deutschen Archäologischen Instituts. Athenische Abteilung 98, p. 265-269; id. (1985), « Tobacco Pipes of Corinth and of the Athenian Agora ", Hesperia 54/2, p. 153-157. Cf. HAYES J.W. (1992), Excavations at Saraçhane in Istanbul, 2 vol., vol. II: The Pottery, Princeton University Press and Dumbarton Oaks Research Library and Collection, Princeton/Washington (D.C.), p. 391-394. S'agissant de la couleur de l'argile qui, pour les pipes ottomanes, constitue un des indices de datation, nous avons eu recours aux travaux d'Amir Dekkel, qui a analysé le matériel recueilli à Baniyas, DeKkel A. (2008), « The Ottoman Clay Pipes », in Tzaferis V. et Israeli Sh. (éds.), Paneas, 3 vol., vol. II : Small Finds and other Studies, (IAA Reports, 38) Israel Antiquity Authority, Jerusalem, p. 124. Quant à la datation, nous avons pris en compte certaines analogies fournies par le matériel provenant d'autres sites archéologiques fouillés sur le territoire de l'Empire ottoman.

${ }^{34}$ Long. - longueur; haut. - hauteur; diam. t. - diamètre intérieur de la tige ; + - dimension du fragment préservé.
} 
26. Pipe à bol sphérique, avec bague biconique (pl. IV 26) Inv. no JM-P-0104

Long. 4,7 cm + ; haut. 3,4 cm +; diam. t. $0,7 \mathrm{~cm}$. La bague est décorée à la roulette et estampé. Le bol porte une décoration estampée formée de rhomboïdes dans lesquels sont inscrits des ronds convexes. Au bas, lignes incisées formant un chevron. Du fourneau il ne reste qu'un petit fragment. Argile blanche (White Page $8 / \mathrm{N}$ ); absence d'inclusions; point de porosité. Datation: XVII ${ }^{\mathrm{e}}$ ou XVIII ${ }^{\mathrm{e}}$ siècle.

\section{Pipe à bol sphérique (pl. IV 27)}

Inv. no JM-P-0092

Long. 3,9 $\mathrm{cm}+$; haut. 2,1 $\mathrm{cm}+$; diam. t. Env. 0,6 $\mathrm{cm}+$. Bol à décor estampé sous forme de lignes ondulées avec, en dessous, un décor de chevrons. Le haut du bol ainsi que l'extrémité de la tige sont cassés. Argile blanche (White Page 8/N); grande quantité d'inclusions noires jusqu'à $0,04 \mathrm{~cm}$. Datation probable: XVII ${ }^{\mathrm{e}}$ ou XVIII ${ }^{\mathrm{e}}$ siècle.

28. Pipe à bol sphérique, bord cassé (pl. IV 28)

Inv. $n^{\circ}$ JM-P-0083

Long. 5,5 cm; haut. 2,5 cm +; diam. t. 0,9 cm. Décor de chevrons à la roulette en dessous du bol. Fleur estampé sur les deux côtés du bol. Devant la bague lignes continues incisées. La bague décorée d'incisions transversales. Argile vert gris (gley $25 / 10 \mathrm{G})$, surface polie. Faible porosité $(0,02 \mathrm{~cm})$; faible quantité d'inclusions blanches. Datation très probable: $\mathrm{XVII}^{\mathrm{e}}$ ou XVIII ${ }^{\mathrm{e}}$ siècle.

\section{Pipe à bol sphérique aplati avec un fourneau fragmentaire (pl. IV 29)} Inv. $n^{\circ}$ JM-P-0086

Long. 5,2 cm; haut. 2,4 cm +; diam. t. 0,9 cm. Une large bague conique occupe la moitié de la longueur de la tige terminée par un anneau conique. En dessous du bol, double chevron réalisé à la roulette; des deux côtés losanges inscrits dans des cercles avec, au-dessus, des rosettes estampées. Le bord du bol cassé. Couleur de la pâte à la cassure: extérieur gris (2.5 Y 6/1); noyau gris bleu foncé (gley 2 4/10B). Absence de porosité, grande quantité d'inclusions calcaires (jusqu'à $0,12 \mathrm{~cm}$ ). Datation: XVII ${ }^{\mathrm{e}}$ ou XVIII ${ }^{\mathrm{e}}$ siècle. 


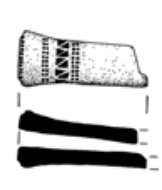

24

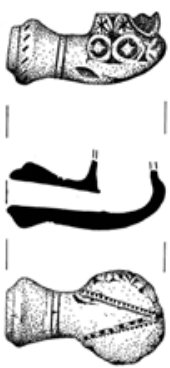

29

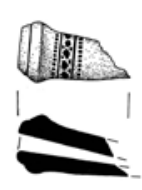

25

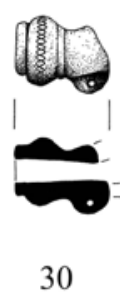

30
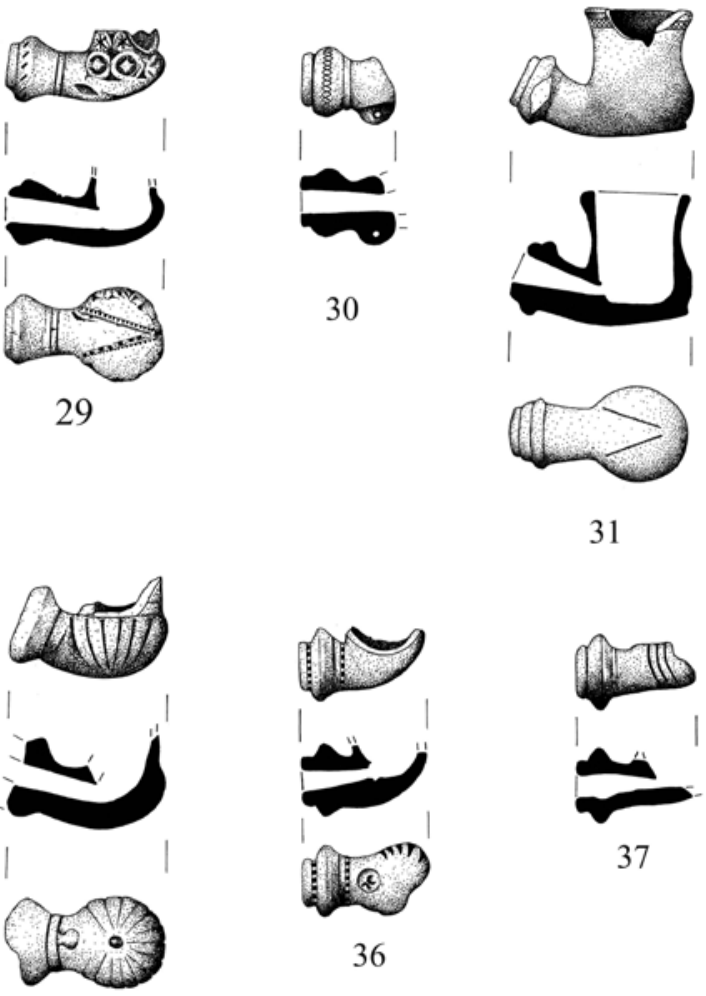

35
31

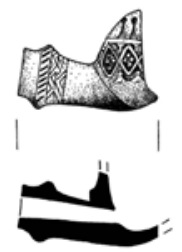

26

27

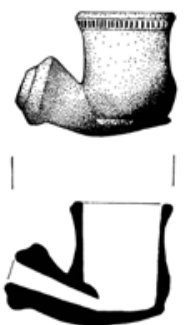

32

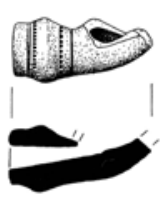

38
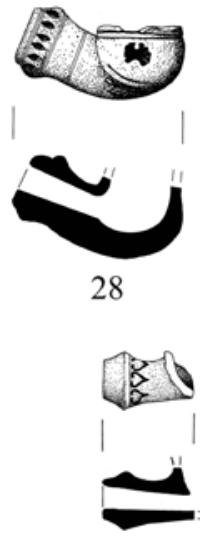

33

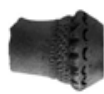

34
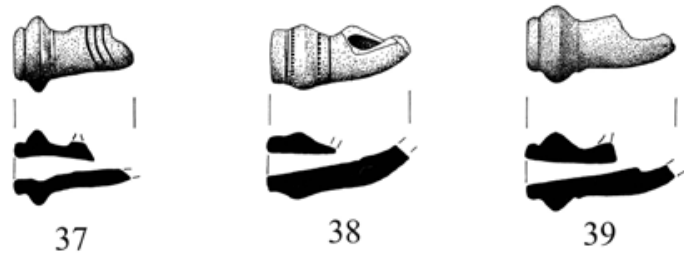

39
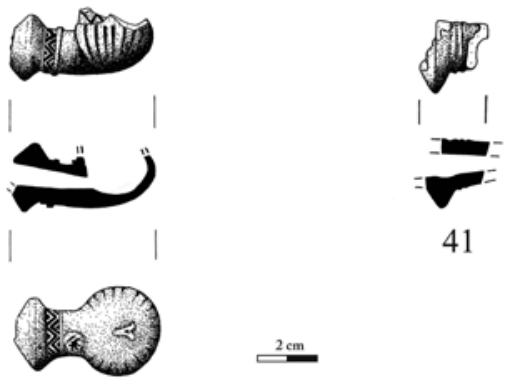

40

Pl. IV - Pipes ottomanes. 
30. Pipe à bague arrondie, terminée par un anneau conique, munie d'une petite préhension ( $\mathrm{pl}$. IV 30)

Inv. n' JM-P-0079

Long. 3,2 cm +; haut. 2,3 cm +; diam. t. 0,8 cm. Bague décorée à la roulette, lignes ondulées entrecroisées. Des deux côtés de la bague des traits réalisés à la roulette. Tige partiellement conservée. Argile grise (gley 1 5/N). Faible porosité, inclusions noires (jusqu'à $0,02 \mathrm{~cm}$ ). Les préhensions sont notées rarement dans les pipes ottomanes. La préhension apparaît sur une pipe de Jérusalem et sur des pipes de Baniyas datées $d u X \mathrm{XII}^{\mathrm{e}}$ et du début du XVIII ${ }^{\mathrm{e}}$ siècle. ${ }^{35} \mathrm{Vu}$ la couleur de l'argile, l'anneau conique à l'extrémité de la tige et le petit diamètre de l'intérieur de la tige, la pipe (cat. $\mathrm{n}^{\circ} 30$ ) semble pouvoir être attribuée à la même période.

\section{Pipe à bol arrondi avec petit disque au bas du bol; fourneau haut (pl. IV 31)}

Sans n ${ }^{\circ}$ 'inv.

Long. 5,6 cm ; haut. 4,4 cm; diam. t. 0,9 cm. Section du fourneau peu typique : partie inférieure bombée, parois concaves, bord évasé. Bague arrondie très simple. Tige terminée par un anneau conique. Décor de carreaux sur le bord; chevron incisé sur le dessous du bol. Ébréchures sur le bord du fourneau et sur la bague. Argile grise (2.5 Y 6/1). Surface recouverte d'engobe de couleur non unie, du rouge brun (2.5 YR $5 / 4)$ au rouge foncé (7.5 R 3/3). Absence de porosité; inclusions noires (jusqu'à $0,15 \mathrm{~cm}$ ), inclusions blanches (jusqu'à $0,02 \mathrm{~cm}$ ). Mis à part la pipe cat. $\mathrm{n}^{\mathrm{o}} 32$, il n'existe point d'analogies parmi les pipes publiées. Il s'agit peut-être d'un modèle de transition entre les pipes à bol sphérique et celles à disque. Compte tenu du diamètre de l'ouverture de la tige, de la couleur de l'argile et de la forme de l'extrémité de la tige, la pipe doit être datée de la fin du XVII ${ }^{\mathrm{e}}$ ou du XVIII ${ }^{\mathrm{e}}$ siècle.

\section{Pipe à petit disque au bas du bol (pl. IV 32)} Inv. $n^{\circ}$ JM-P-0089

Long. 5,5 cm; haut. 4,3 cm; diam. t. 0,75 cm. Tige terminée par un anneau conique; bague très simple. Décor à la roulette sur le bord du fourneau; un chevron incisé sur le dessous du bol. Ébréchures sur l'extrémité de la tige et sur le bord. Argile

\footnotetext{
35 Avner R. et De'Adle T. (2009), «Jerusalem, the Ohel Yizhaq Syngogue. Final report », Hadashot Arkheologiyot 121, fig. 4.1; cf. DekKel, «The Ottoman Clay Pipes », p. 131, $\mathrm{n}^{\text {os }}$ 1, 6, 20, 23. Les Pipes à préhensions sont connues aussi du nord du Sinaï. Celles-ci sont datées aussi du XVII ${ }^{\mathrm{e}}$ et du début du $\mathrm{XVIII}^{\mathrm{e}}$ siècles : SAIDEL B.A. (2008), « Smoking out Ottoman Sites in Northern Sinai, Egypt: The Use of Clay Tobacco Pipes for Identifying the Nature of Settlements in the Ottoman Period », Palestine Exploration Quarterly 140/1, p. 60, fig. 3.2, 3 .
} 
gris foncé (2.5 Y 4.1), engobe rouge brun (5 YR 5.4). Inclusions blanches (jusqu'à $0,04 \mathrm{~cm}$ ); inclusions noires (jusqu'à $0,03 \mathrm{~cm}$ ). La pipe cat. $\mathrm{n}^{\circ} 32$ représente le même type que le cat. $n^{\circ} 31$.

\section{Pipe à bague biconique (pl. IV 33)}

Inv. n JM-P-0099

Long. 3,1 cm +; haut. 1,7 cm +; diam. t. 0,75 cm. Absence d'embout distinct à l'extrémité de la tige. Sur la bague un décor estampé. De toute la pipe il ne subsiste que la tige. Argile grise (7.5 YR 6/1). Absence de porosité; absence d'inclusions. Datation indéterminée.

\section{Fragment de pipe (pl. IV 34)}

Inv. $\mathrm{n}^{\circ} \mathrm{JM}-\mathrm{P}-0096$

Long. 3,1 cm +; haut. 2,7 cm +, diam. t. 1,3 cm. Il ne subsiste qu'un fragment de tige avec une bague conique décorée d'arceaux estampés. Derrière la bague, un décor à la roulette. Absence d'anneau conique à l'extrémité de la tige. Argile grise, engobe rouge foncé ( $7.5 \mathrm{R} 3 / 4)$. Moyenne quantité de petites inclusions noires et blanches. Vu l'absence d'anneau conique à l'extrémité de la tige, la couleur rouge de l'engobe et le diamètre intérieur de la tige supérieur à $1 \mathrm{~cm}$, la pipe doit être datée du XVIII ${ }^{\mathrm{e}}$ siècle, voire de la période postérieure. Les pipes découvertes à Babadag en Dobroudja, qui présentent des bagues aux formes et aux décors similaires, sont attribuées aux XVIII ${ }^{\mathrm{e}}$ et XIX ${ }^{\mathrm{e}}$ siècles. $^{36}$

\section{Pipe à bol arrondi, fourneau cylindrique dans sa partie inférieure (pl. IV 35)} Inv. $\mathrm{n}^{0} \mathrm{JM}-\mathrm{P}-0084$

Long. 4,9 cm +; haut. 3,3 cm +; diam. t. Env. 0,7 cm +. Bague biconique. L'embout était sans doute en forme d'anneau conique. Le dessous du bol porte un décor de conque incisé; sur la tige, devant la bague, figurent des anneaux. Sur la tige, un motif rond estampé illisible. Bord du fourneau et extrémité de la tige ébréchés. Argile gris rosâtre (7.5 YR 7/2). Quantité moyenne d'inclusions de mica (jusqu'à 0,01 cm), faible porosité (jusqu'à $0,04 \mathrm{~cm}$ ). Il est possible d'évoquer comme analogues les pipes cat. ${ }^{\text {os }}$ 36-41. La pipe de Jerash présente la même forme mais elle est couverte d'engobe rouge ${ }^{37}$. Des formes similaires proviennent aussi de Baniyas, mais elles

\footnotetext{
${ }^{36}$ Costea I., Stănică A. et Ignat A. (2007), « Pipe de lut descoperite la Babadag », Peuce 5, nºs 21, 23-25, p. 342, 343, pl. 4. 1, 2-5.

${ }^{37}$ Clank V. A., Bowsher J. M. C. et Stewart J. D. (1986), « The Jerash North Theatre. Architecture and Archaeology 1982-1983 », in ZAYADINE F. (éd.), Jerash Archaeological Project 1981-1983, I, Department
} 
aussi sont couvertes d'engobe ${ }^{38}$. Compte tenu de la typologie de Rebecca Robinson et des analogies mentionnées, la pipe peut être datée du XVII ${ }^{\mathrm{e}}$ ou du XVIII ${ }^{\mathrm{e}}$ siècle.

\section{Pipe à bol arrondi avec une haute bague biconique (pl. IV 36)} Inv. no JM-P-0087

Long. 4,7 cm +; haut. 2,1 cm +, diam. t. 0,7 cm. Extrémité de la tige en forme d'anneau conique. Bol partiellement cassé. Sur le dessous de la tige, un motif rond estampé, à peine visible. Devant et derrière la bague un décor à la roulette; sur le dessous du bol, des lignes incisées imitant la coquille. Argile grise (2.5 Y 5/1). Moyenne quantité d'inclusions blanches et faible quantité d'inclusions noires, faible porosité (pores oblongs). Cette pipe représente le même type que la pipe cat. $\mathrm{n}^{\circ} 35$.

\section{Pipe à bague biconique délimitée des deux côtés par un décor à la roulette (pl. IV 37) \\ Inv. $n^{\circ}$ JM-P-0091}

Long. 3,9 cm + ; haut. 2,9 cm +; diam. t. $0,7 \mathrm{~cm}$. Tige terminée par un anneau conique. Sur le dessous de la tige un décor estampé de quatre cercles irrégulièrement disposés. Sur le dessous du bol des lignes incisées imitant la coquille. Bol très fragmentaire. Argile grise (2.5 Y 5/1). Faible quantité d'inclusions noires (jusqu'à $0,1 \mathrm{~cm}$ ). Cette pipe représente le même type que les pipes cat. $\mathrm{n}^{\text {os }} 35$ et 36 .

\section{Pipe avec bague légèrement arrondie (pl. IV 38)}

Inv. no JM-P-0097

Long. 4,7 cm +; haut. 1,8 cm +, diam. t. 0,8 cm. Tige terminée par un anneau conique. La tige porte un décor à la roulette. Sur le dessous du bol des lignes incisées imitant la coquille. Manque une grande partie du fourneau. Argile grise (gley 1 5/N), sans porosité ni inclusions. Forme très proche des cat. ${ }^{\text {os }} 35,36$ et 37.

\section{Pipe à bol arrondi avec une bague biconique aplatie (pl. IV 39)} Inv. n' JM-P-0098

Long. 5,1 cm +; haut. 2,6 cm +; diam. t. 0,9 cm. Tige terminée par un anneau conique. Sur le dessous de la tige un motif rond estampé avec une rosette à cinq

of Antiquities of Jordan, Amman, p. 266, pipe B, pl. XXVII.2.

${ }^{38}$ DekKel, «The Ottoman Clay Pipes », p. 142, $n^{\text {os }} 46,47$, fig. 4.9. La pipe d'Ayas (Turquie) présente une forme et une couleur semblables. Seul le décor de la bague est différent: EsER E. (2009), « From the Roman Settlement of Aigai to Ayas », Vjesnik Arheoloskog muzeja u Zagrebu 18, p. 434, fig. 19. 
pétales à peine visible. Entre la bague et la rosette un décor à la roulette. Sur le dessous du bol des lignes incisées imitant la coquille. De tout l'objet il ne subsiste que la tige et un fragment de bol. Argile gris foncé ( $2.5 \mathrm{Y} 4 / 1$ ). Inclusions blanches (jusqu'à $0,01 \mathrm{~cm})$. La pipe représente le même type que les pipes cat. $\mathrm{n}^{\mathrm{os}} 35,36,37$ et 38 .

\section{Pipe à bol arrondi avec une bague biconique (pl. IV 40)} Inv. n JM-P-0106

Long. 4,8 cm +; haut. 2,3 cm +; diam. t. en dessous de $1 \mathrm{~cm}$. Embout de la tige en forme d'anneau conique aujourd'hui inexistant. Sur le dessous de la tige un décor estampé à peine visible. Sur le dessous du bol des lignes incisées imitant la coquille. La tige décorée à la roulette et estampée à proximité de la bague. Le bord du bol cassé. Argile grise, engobe rouge foncé (10 R 3/3) partiellement préservé. Inclusions noires (jusqu'à $0,03 \mathrm{~cm}$ ). Porosité moyenne (jusqu'à $0,02 \mathrm{~cm}$ ). La pipe cat. $\mathrm{n}^{\circ} 41$ présente une bague et un engobe semblables. Cette pipe est proche par sa forme des cat. $\mathrm{n}^{\text {os }} 35,36$ et 37 .

\section{Pipe à bague biconique et tige partiellement préservée (pl. IV 41)} Inv. n JM-P-0100

Long. $2,1 \mathrm{~cm} \mathrm{+;} \mathrm{haut.} 2,3 \mathrm{~cm}+$; diam. t. env. $0,8 \mathrm{~cm}$. La tige était très vraisemblablement terminée par un anneau conique. Sur le pourtour de la tige un décor à la roulette. Argile gris clair (5Y 7/1), engobe rougeâtre (5 R 4/2). Absence de porosité, faible quantité d'inclusions noires (jusqu'à $0,02 \mathrm{~cm}$ ). La pipe cat. ${ }^{\circ} 41$ présente une bague et un engobe semblables.

42. Pipe à bol arrondi, assez bas, et à double registre sur les parois du fourneau (pl. V 42) Inv. $n^{\circ}$ JM-P-0093

Long. $5,2 \mathrm{~cm}$; haut. $5,1 \mathrm{~cm}+$. Bague très fragmentaire. Le registre supérieur porte un décor de carreaux à la roulette qui, sur la tige et sur la bague, mais aussi sur le dessous du bol, est accompagné de lignes incisées, l'ensemble formant un double chevron. Le registre inférieur porte un décor côtelé excisé et des feuilles oblongues estampées. Le bol est décoré de figures géométriques estampées. L'embout de la tige cassé, ébréchures de surface, la partie supérieure du bord du fourneau cassée. Argile gris clair (10 YR 7/1). Faible quantité d'inclusions blanches (jusqu'à $0,01 \mathrm{~cm}$ ), porosité moyenne (pores jusqu'à $0,02 \mathrm{~cm}$ ). Datation probable: XVIII ${ }^{\mathrm{e}}$ siècle. 
43. Pipe à bol arrondi, à fourneau cylindrique (pl. V 43) Inv. $n^{\circ}$ JM-P-0103

Long. 3,3 cm +, haut. 4,5 cm +. Décor en forme de lignes incisées verticales sur les parois du fourneau et sur le bol, chevron à la roulette sur le dessous du bol. Grande partie du bol et de la tige cassée. Argile blanche (gley $18 / \mathrm{N}$ ). Inclusions noires (jusqu'à $0,04 \mathrm{~cm}$ ). Des formes semblables de pipes apparaissent dans le matériel archéologique provenant de Baniyas ${ }^{39}$. Datation possible: $\mathrm{XVIII}^{\mathrm{e}}$ siècle.

\section{Pipe à bague en forme de bretzel ( $\mathrm{pl} . \mathrm{V} 44)$}

Inv. no JM-P-0094

Long. 4,7 cm +; haut. 2,7 cm +; diam. t. 0,8 cm. Avec un large anneau conique à l'embout de la tige et un décor à la roulette sur toutes les parties conservées de l'objet. Manque un grand fragment du bol. Couleur de la pâte à la cassure: intérieur gris clair (5 Y 7/1), extérieur rouge clair (2.5 YR 5/8). Forte porosité (jusqu'à $0,1 \mathrm{~cm}$ ), grande quantité d'inclusions calcaires (jusqu'à $0,1 \mathrm{~cm}$ ). Datation très vraisemblable: fin $\mathrm{XVII}^{\mathrm{e}}$ ou XVIII ${ }^{\mathrm{e}}$ siècle.

\section{Tige fragmentaire de pipe avec anneau conique (pl. V 45)} Inv. no JM-P-0095

Long. 2,9 cm +; haut. $2 \mathrm{~cm}+$; diam. t. $0,6 \mathrm{~cm}$. Bague simple et décor à la roulette. Argile jaune rougeâtre (5 YR 7/8), menues inclusions blanches. Datation difficile à établir.

46. Pipe avec large bague arrondie à décor de traits obliques incisés (pl. V 46) Inv. $n^{\circ}$ JM-P-0081

Long. 3,8 cm +; haut. 2,7 cm +; diam. t. env. 0,6 cm. Tige à facettes terminée par un anneau conique. Sur la tige un décor estampé en forme de feuilles de chanvre. Manque le bol et une partie de l'embout de la tige. Argile rouge (2.5 YR 4/8), surface polie. Faible quantité d'inclusions blanches (jusqu'à $0,05 \mathrm{~cm}$ ), absence de porosité. Vu la présence de l'anneau conique sur la tige, la couleur de la pipe ainsi que le décor estampé, la pipe pourrait être attribuée au XVIII ${ }^{\mathrm{e}}$ siècle. Cependant, compte tenu de l'état fragmentaire de l'objet, cette attribution chronologique est incertaine.

\footnotetext{
${ }^{39}$ Dekkel, « The Ottoman Clay Pipes », p. 140, 142, 144, $\mathrm{n}^{\text {os }} 41,44,49$, fig. 4.8, 4.9.
} 


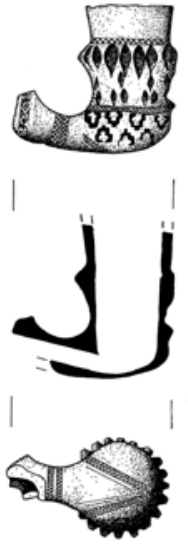

42

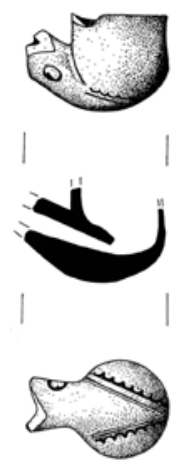

48

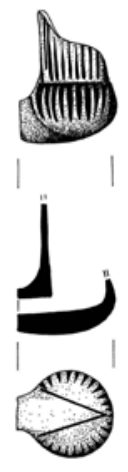

43

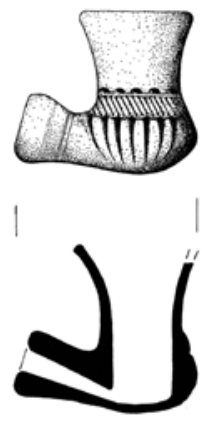

49
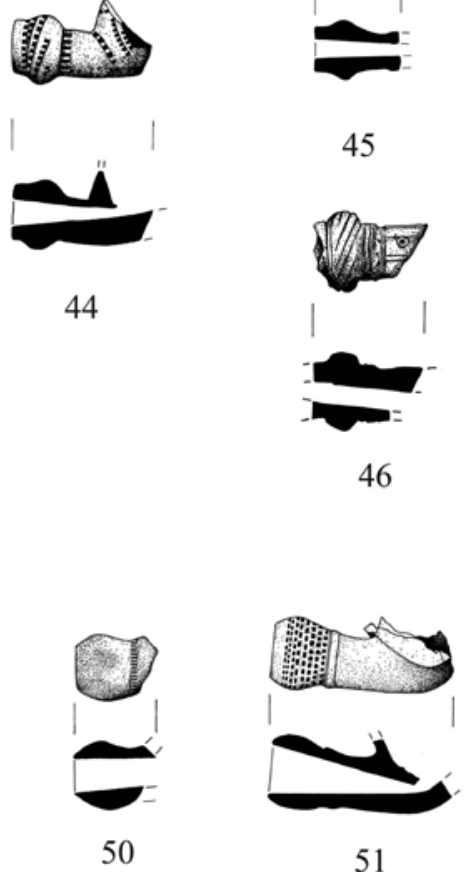

51

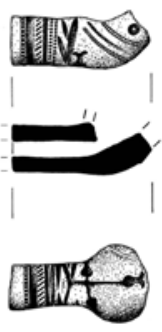

47

46

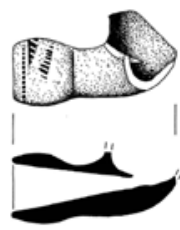

52

Pl. V - Pipes ottomanes. 
47. Pipe sans bague (ou bague cassée) (pl. V 47) Inv. $n^{\circ}$ JM-P-0082

Long. 4,9 cm + ; haut. 1,9 cm +; diam. t. env. 0,5 cm +. Décor à la roulette sur la tige et sur le dessous du bol où il prend la forme de chevrons. En outre, sur le dessous du bol, un décor estampé en forme de grenades. Sur la paroi droite du bol, un cercle estampé partiellement effacé. Manquent l'embout de la tige et un grand fragment du bol. Argile rouge (2.5 YR 4 juin), surface polie. Absence de porosité, grande quantité d'inclusions calcaires (jusqu'à $0,02 \mathrm{~cm}$ ). Datation incertaine. La couleur de l'argile proche de celle de la pipe cat. $n^{\circ} 46$.

\section{Pipe à bol arrondi et à fourneau partiellement conservé (pl. V 48)} Inv. no JM-P-0085

Long. 4,5 cm +; haut. 3,3 cm +. Sur le dessous de la tige, à proximité du bol, un cercle estampé peu visible. Manquent l'embout de la tige et le bord du bol. Sur le dessous du bol, un décor en forme de deux chevrons dont l'un est incisé et l'autre estampé en arceaux. Couleur de la pâte à la cassure: intérieur gris (5 YR 5/1), extérieur rouge clair (10 $\mathrm{R} 7 / 6)$. Grande quantité d'inclusions noires (jusqu'à $0,02 \mathrm{~cm}$ ) et d'inclusions calcaires (jusqu'à $0,02 \mathrm{~cm}$ ). Datation très probable: XVIII ${ }^{\mathrm{e}}$ ou XIX ${ }^{\mathrm{e}}$ siècle.

\section{Pipe à bol arrondi et aux parois du fourneau évasées (pl. V 49)} Inv. $n^{\circ}$ JM-P-0107

Long. 5,7 cm; haut. 5,4 cm; diam. t. 0,7 cm. Large bague arrondie. Absence d'anneau conique à l'extrémité de la tige. Décor estampé sur le bol; sur le dessous du bol, décor incisé de traits qui ressemblent à une coquille. Sur la tige un décor d'anneaux incisés. Objets conservé presque intact; petites ébréchures sur le bord et sur l'extrémité de la tige. Argile gris vert clair (gley $18 / 10 \mathrm{Y}$ ), engobe jaune rougeâtre (5 YR 6/6). Quantité moyenne d'inclusions noires (jusqu'à $0,01 \mathrm{~cm}$ ), porosité moyenne. Comme analogies les plus proches, il faut évoquer les pipes de Beyrouth ${ }^{40}$ datant du $\mathrm{XIX}^{\mathrm{e}}$ siècle. Les clichés de cette pipe se trouvent dans les enveloppes 14 PCMA et $48 \mathrm{PCMA}^{41}$ des archives photographiques de Jean Mécérian.

\footnotetext{
${ }^{40}$ Bartl K. (2003), « Clay Pipes from Ottoman Beirut », Bulletin d'Archéologie et d'Architecture Libanaises 7 , p. $337, \mathrm{n}^{\text {os }} 6,7,8$, pl. 2.

${ }^{41}$ Le contenu de l'enveloppe 14 PCMA est présenté dans la note accompagnant la description de la bouteille, cat. 23. L'enveloppe 48 PCMA contient les photos du monastère de Saint-Syméon-Stylite-le-Jeune, qu'il s'agisse des photos des reliefs appartenant à la collection étudiée ( $c f$. l'article de Dagmara Wielgosz dans le présent volume), ou de celles des reliefs dont la provenance et le lieu actuel de conservation sont inconnus. Au verso de certains clichés figure la date 1938 inscrite au crayon.
} 
50. Pipe à bague arrondie sans embout en forme d'anneau conique (pl. V 50) Inv. no JM-P-0105

Long. 2,8 cm +; haut. 2,4 cm +; diam. t. 1,1 cm. Tige décorée à la roulette. Tige fragmentaire. Couleur de la pâte à la cassure : intérieur gris foncé (gley $13 / \mathrm{N}$ ), extérieur rose (5 YR 7/3); engobe rouge (7.5 R 4/6). Faible porosité (jusqu'à 0,01 cm); petite quantité d'inclusions blanches (jusqu'à $0,02 \mathrm{~cm}$ ). Vu le diamètre intérieur de la tige, l'absence d'anneau conique à l'extrémité de la tige, la couleur de la pâte ainsi que la présence d'engobe, il faut dater la pipe du XVIII ${ }^{\mathrm{e}}$ ou XIX ${ }^{\mathrm{e}}$ siècle.

51. Très vraisemblablement une pipe en forme de lys avec bague arrondie (pl. V 51) Inv. $\mathrm{n}^{\circ} \mathrm{JM}-\mathrm{P}-0080$

Long. 6,2 cm +; haut. 2,6 cm +; diam. t. 1,6 cm. Absence d'anneau conique à l'extrémité de la tige. Décor estampé en forme de petits rectangles sur la bague, lignes incisées devant la bague, deux traits incisés en forme de chevron sur le dessous du bol. Bol très fragmentaire. Argile brun rougeâtre ( 5 YR 5/4); surface polie. Forte porosité (jusqu'à $0,02 \mathrm{~cm}$ ). Comme analogies, il faut signaler la pipe cat. $\mathrm{n}^{\circ} 52$ et les pipes de Kerem Maharal, 'Atlit, Tel Mevorakh et Zir'in ${ }^{42}$. D'après les analogies, vu la forme de l'embout de la tige et le diamètre de l'ouverture, il faut dater la pipe de la fin du XIX ${ }^{\mathrm{e}}$ ou du début du $\mathrm{XX}^{\mathrm{e}}$ siècle.

\section{Pipe à bol sphérique avec une large bague arrondie et aplatie ( $\mathrm{pl}$. V 52)} Inv. $\mathrm{n}^{\mathrm{o}} \mathrm{JM}-\mathrm{P}-0102$

Long. 5,6 cm +; haut. $3 \mathrm{~cm}+$; diam. t. 1,6 cm. Absence d'anneau conique à l'extrémité de la tige. Décor à la roulette sur la bague. Couleur de la pâte à la cassure: intérieur gris foncé (gley $14 / \mathrm{N}$ ), extérieur rouge (10 R 5/6). Faible porosité, petite quantité d'inclusions blanches (jusqu'à $0,03 \mathrm{~cm}$ ). La pipe ressemble par sa forme à la pipe cat. $\mathrm{n}^{\circ} 51$.

\footnotetext{
42 Oren E. (2010), « Kerem Maharal. Final Report », Hadashot Arkheologiyut 122, fig. 4.2; BuchenNino A. (2010), «'Atlit Harbor. Final Report», Hadashot Arkeologiyot 122, fig. 4.18; SA ‘̄ì 'A. (2009), « Tel Mevorakh. Final Report », Hadashot Arkheologiyot 121, fig. 1.11; SimPSON J. (2002), «Ottoman Pipes from Zir'in (Tell Jezreel) », Levant 34, p. 164-165, figs 1.7-8, 2.9; SAIDEL, « Smoking out Ottoman Sites », p. 60, fig. 3.14. Tous ces objets sont datés de la fin de la période ottomane ( $\mathrm{XIX}^{\mathrm{e}}$ et début $\mathrm{XX}^{\mathrm{e}}$ siècles).
} 


\section{BIBLIOGRAPHIE}

Alpago-Novello A., Beridze V. et Lafontaine-Dosogne J. (1980), Art and Architecture in Medieval Georgia, (Publications d'histoire de l'art et d'archéologie de l'Université Catholique de Louvain, 21) Inst. Sup. d'Archéologie et d'Histoire de l'art, Louvain-La-Neuve.

Anderson-Stojanović V. R. (1987), « The Chronology and Function of Ceramic Unguentaria », American Journal of Archaeology 91/1, p. 105-122.

D'ANgela C. (1995), «Recenti scoperte paleocristiane ad Otranto », Corso di cultura sull'arte ravennate e bizantina 42 , p. 275-288.

Avner R. et De'AdLE T. (2009), « Jerusalem, the Ohel Yizhaq Syngogue. Final report », Hadashot Arkheologiyot 121 (version électronique non paginée).

Bagatti B. (1972), «Il cristianesimo ad Eleuteropoli (Beir Gebrin)», Liber Annuus 22, p. 109-129.

Bartl K. (2003), « Clay Pipes from Ottoman Beirut », Bulletin d'Archéologie et d'Architecture Libanaises 7, p. 321-340.

Buchennino A. (2010), «'Atlit Harbor. Final Report», Hadashot Arkeologiyot 122 (version électronique non paginée).

Butler A. J. (1884), The Ancient Coptic Churches of Egypt, 2 vol., Clarendon Press, Oxford.

Camber R. (1979), « A Hoard of Terracotta Amulets from the Holy Land », in Actes du XV congrès international d'études byzantines, Athènes septembre 1976, Association internationale des études byzantines, Athènes, p. 99-106.

Canivet M.-T. (1978), « Le reliquaire à huile de la grande église de Huarte (Syrie) », Syria 55/12, p. 153-162.

Clark V. A., Bowsher J. M. C. et Stewart J. D. (1986), « The Jerash North Theatre. Architecture and Archaeology 1982-1983 », in Zayadine F. (éd.), Jerash Archaeological Project 19811983, I, Department of Antiquities of Jordan, Amman, p. 205-270.

Costea I., Stănică A. et Ignat A. (2007), «Pipe de lut descoperite la Babadag », Peuce 5, p. 335-362.

Cotsonis J. A. (1994), Byzantine Figural Processional Crosses: Catalogue of an Exhibition at Dumbarton Oaks, 23 September 1994 - 29 January 1995, (Dumbarton Oaks Byzantine Collection Publications, 10) Dumbarton Oaks Research Library and Collections, Washington (D.C.).

Dalton O. M. (1901), Catalogue of Early Christian Antiquities and Objects from the Christian East in the Department of British and Mediaeval Antiquities and Ethnography of the British Museum, British Museum, Londres.

Dauterman Maguire E., Maguire H. P. and Duncan-Flowers M. J. (1989), Art and Holy Powers in the Early Christian House, (Illinois Byzantine Studies, 2) University of Illinois Press, Urbana.

Dekrel A. (2008), « The Ottoman Clay Pipes », in Tzaferis V. and Israeli Sh. (éds.), Paneas, 3 vol., vol. II : Small Finds and other Studies, (IAA Reports, 38) Israel Antiquity Authority, Jerusalem, p. 113-164.

Djobadze W. (1986), Archaeological Investigations in the Region West of Antioch on-the-Orontes, (Forschungen zur Kunstgeschichte und christlichen Archäologie, 13) Franz Steiner Verlag, Wiesbaden.

Donceel-Voûte P. (1995), "Le rôle des reliquaires dans les pèlerinages », in Dassman E. et Engemann J. (éds.), Akten des XII. Internationalen Kongresses für christliche Archäologie, Bonn 22.-28. September 1991, 2 vol., (Jahrbuch für Antike und Christentum 
Ergänzungsband, 20), Aschendorffsche Verlagsbuchhandlung and Pontificio Istituto di archeologia cristiano, Münster/Cité du Vatican, vol. I, p. 184-205.

ElBERN V. H. (1965), « Eine frühbyzantinische Reliefdarstellung des älteren Symeon Stylites », Jahrbuch des Deutschen Archäologischen Instituts 80, p. 280-304.

Eser E. (2009), «From the Roman Settlement of Aigai to Ayas », Vjesnik Arheoloskog muzeja u Zagrebu 18, p. 421-441.

Fansa M. et Bollmann B. (éds.) (2008), Die Kunst der frühen Christen in Syrien. Zeichen, Bilder und Symbole vom 4. bis 7. Jahrhundert. Begleitband zur Sonderausstellung im Landesmuseum Natur und Mensch Oldenburg, (Schriftenreihe des Landesmuseums Natur und Mensch, 60) Verlag Philipp von Zabern, Mainz am Rhein.

FernandeZ R. (1975), « Le culte et l'iconographie des stylites », in Peña I., Castellana P. et Fernandez R. (éds.), Les Stylites syriens, (Studium Biblicum Franciscanum, Collection minor, 16) Franciscan Printing Press, Milan, p. 163-217.

Id. (1985), «Les représentations des stylites », Studia Orientalia Christiana, Collectanea 18, p. $115-151$.

GeSSEL W. (1988), « Das Öl der Märtyrer. Zur Funktion und Interpretation der Ölsarkophage von Apamea in Syrien », Oriens Christianus 72, p. 183-202.

Gilli M. (2002), Le ampolle di San Mena. Religiosità, cultura materiale e sistema produttivo, (Tardoantico e Medioevo - Studi e strumenti di archeologia, 5) Palombi, Rome.

GöкÇAY M. et Asal R. (2008), « Excavations in Küçük Ayasofya Street, Eminönü, Istanbul », Anatolia Antiqua 16, p. 207-230.

Grabar A. (1950), « Quelques reliquaires de saint Démétrios et le martyrium du saint à Salonique », Dumbarton Oaks Papers 5, p. 3-28.

Hayes J.W. (1992), Excavations at Saraçhane in Istanbul, 2 vol., vol. II: The Pottery, Princeton University Press and Dumbarton Oaks Research Library and Collection, Princeton/Washington (D.C.).

Hillbom N. (2000-2001), « Minoan and Eastern Mediterranean Games and Game Boards: A History of Research », Opuscula Atheniensia 25-26, p. 53-65.

Jolivet-Lévy C. (1998), « Note sur la représentation des archanges en costume impérial dans l'iconographie byzantine », Cahiers Archéologiques 46, p. 121-128.

Kalavrezou-MaXeiner I. (1985), Byzantine Icons in Steatite, 2 vol., (Byzantina Vindobonensia, 15) Verlag der Österreichischen Akademie der Wissenschaften, Vienne.

Kiss Z. (1989), Les ampoules de Saint Ménas découvertes à Kôm el-Dikka (1961-1981), (Alexandrie, 5) Państwowe Wydawnictwo Naukowe, Varsovie.

LAFLI E. (2003a), Studien zu hellenistischen, kaiserzeitlichen und spätantik-frühbyzantinischen Tonunguentarien aus Kilikien und Pisidien (Südtürkei): der Forschungsstand und eine Auswahl von Fundobjekten aus den örtlichen Museen, Universität zu Köln, (thèse de doctorat inédite).

Id. (2003b), «Hellenistische, römischkaiserzeitliche und spätantik-frühbyzantinische Tongunguentarien aus dem Museum von Gaziantep (Südosttürkei) », Instrumentum 18 (December), p. 31-34.

LAFONTAINe-Dosogne J. (1967), Itinéraires archéologiques dans la région d'Antioche: Recherches sur le monastère et sur l'iconographie de S. Syméon Stylite le Jeune (Bibliothèque de Byzantion, 4) Édition de Byzantion, Bruxelles.

Id. (1981), « Une eulogie inédite de St Syméon Stylite le Jeune », Byzantion 51/2, p. 631-634.

Lambert Ch. et Pedemonte Demeglio P. (1994), « Ampolle devozionali ed itinerary di pellegrinaggio tra IV e VII secolo », Antiquité tardive 2, p. 205-231. 
Lassus J. (1932), «Images de stylites », Bulletin d'études orientales 2, p. 67-82.

VAN Lohuizen-Mulder M. (1988), «The Cathedra of St. Mark in Venice», Bulletin Antieke Beschaving 63, p. 165-179.

Mango M. M. (1986), Silver from Early Byzantium. The Kaper Koraon and Related Treasures, Walters Art Gallery, Baltimore.

McNicoll A.W. et al. (1992), Pella in Jordan 2. The Second Interim Report of the Joint University of Sydney and College of Wooster Excavations at Pella, 1982-1985, (Mediterranean Archaeology Supplement, 2) Meditarch, Sydney.

MÉCÉRIAN J. (1934), «Une mission archéologique dans l'Antiochène. Rapport sur la deuxième campagne de fouilles $1933 »$, Comptes rendus des Séances de l'Académie des Inscriptions et Belles-Lettres 78/2, p. 144-149.

Id. (1948), « Monastère de Saint-Siméon-Stylite-le-Jeune, exposé des fouilles », Comptes rendus des Séances de l'Académie des Inscriptions et Belles-Lettres 92/3, p. 323-328.

Id. (1951), «Le monastère de Saint Syméon le Stylite du Mont Admirable », in Actes du $V I^{e}$ Congrès international des études byzantines, Paris 27 juillet -2 août 1948, 2 vol. en 1, École des Hautes Études, Paris, vol. II, p. 299-302.

Id. (1962), «Les inscriptions du Mont Admirable», Mélanges de l'Université Saint-Joseph 38, p. $297-330$.

Id. (1964), «Expédition archéologique dans l'Antiochène occidentale », MUSJ 40, p. 1-144.

Merlat P. (1949), « Nouvelles images de Saint Syméon le Jeune », in Mélanges d'archéologie et d'histoire offerts à Charles Picard à l'occasion de son $65^{e}$ anniversaire, 2 vol. (= Revue archéologique, 6. sér., t. 29-32), PUF, Paris, vol. II, p. 720-731.

Metzger C. (1981), Les ampoules à eulogie du Musée du Louvre, (Notes et documents des musées de France, 3) Éditions de la Réunion des musées nationaux, Paris.

Millet G. (1933), «La mission archéologique du P. Mécérian dans l'Antiochène », Comptes rendus des Séances de l'Académie des Inscriptions et Belles-Lettres 77/3, p. 343-348.

Id. (1935), «Un rapport du R.P. Mécérian sur les fouilles au monastère de Saint-Syméon-le Jeune au Mont Admirable (Syrie) », Comptes rendus des Séances de l'Académie des Inscriptions et Belles-Lettres 79/2, p. 195-197.

Id. (1936), «Rapport du P. Mécérian sur les fouilles au monastère de Saint-Syméon le Jeune au Mont Admirable (Syrie), Comptes rendus des Séances de l'Académie des Inscriptions et Belles-Lettres 80/3, p. 205-206.

Mouterde R. (1947), « Nouvelles images de stylites », Orientalia Christiana Periodica 13, p. $245-250$.

Oren E. (2010), « Kerem Maharal. Final Report », Hadashot Arkheologiyut 122 (version électronique non paginée).

PeñA I. (2000), Lieux de pèlerinage en Syrie, (Studium Biblicum Franciscanum, Collectio Minor, 38) Franciscan Printing Press, Milan.

PIERI D. (2009), «Saint-Syméon-le-stylite (Syrie du Nord): Les bâtiments d'accueil et les boutiques à l'entrée du sanctuaire », Comptes rendus des Séances de l'Académie des Inscriptions et Belles-Lettres 153/4, p. 1393-1419.

Rahmani L.Y. (1979), « The Adoration of the Magi on Two Sixth-Century C.E. Eulogia Tokens », Israel Exploration Journal 29/1, p. 34-36.

Id. (1993), «Eulogia Tokens from Byzantine Bet She'an », 'Atiqot 22, p. 109-119.

Robinson R. (1983), «Clay Tobacco Pipes from the Kerameikos », Mitteilungen des Deutschen Archäologischen Instituts. Athenische Abteilung 98, p. 265-285.

Id. (1985), « Tobacco Pipes of Corinth and of the Athenian Agora », Hesperia 54/2, p. 149-203. 
SA ‘īD ‘A. (2009), « Tel Mevorakh. Final Report», Hadashot Arkheologiyot 121 (version électronique non paginée).

SaIdel B.A. (2008), « Smoking out Ottoman Sites in Northern Sinai, Egypt: The Use of Clay Tobacco Pipes for Identifying the Nature of Settlements in the Ottoman Period », Palestine Exploration Quarterly 140/1, p. 55-69.

Segal A. et al. (2003), Hippos-Sussita. Fourth Season of Excavations, June - July 2003, Zinman Institute of Archaeology, University of Haifa, Haifa.

Simpson J. (2002), « Ottoman Pipes from Zir'in (Tell Jezreel)», Levant 34, p. 159-172.

Sodini J.-P. (1989), «Remarques sur l'iconographie de Syméon l'Alépin, le premier stylite », Monuments et Mémoires. Fondation Eugène Piot 70, p. 29-53.

Id. (1993), « Nouvelles eulogies de Syméon », in Jolivet-Lévy C., Kaplan M. et Sodini J.-P. (éds.), Les saints et leur sanctuaire à Byzance: textes, images et monuments (Byzantina Sorbonensia, 11) Publication de la Sorbonne, Paris, p. 25-33.

Tchalenko G. (1958), Villages antiques de la Syrie du Nord, le massif du Bélus à l'époque romaine, 3 vol., (Bibliothèque archéologique et historique, 50) Librairie Orientaliste Paul Geuthner, Paris (vol. III).

Ulbert Th. (1990), Der kreuzfahrerzeitliche Silberschatz aus Resafa-Sergiupolis, (Resafa, 3) Philipp von Zabern, Mainz am Rhein.

VIKAN G. (1991), « Guided by Land and Sea. Pilgrim Art and Pilgrim Travel in Early Byzantium », in Dassmann E. et Thraede K. (éds.), Tesserae. Festschrift für Josef Engemann, (Jahrbuch für Antike und Christentum, Ergänzungsband, 18) Aschendorffsche Verlagsbuchhandlung, Münster, p. 74-92.

Volbach W.F. (1966), «Zur Ikonographie des Styliten Symeon des Jüngeren », in Schumacher W.N. (éd.), Tortulae: Studien zu altchristlichen und byzantinischen Monumenten, (Römische Quartalschrift für christliche Altertumskunde und Kirchengeschichte, Supplementheft, 30) Herder, Rome/Freiburg/Vienne, p. 293-299.

De Voogt A. (2010), « Mancala Players at Palmyra », Antiquity 84/326, p. 1055-1066.

Weinberg Davidson G.R. (1952), The Minor Objects, (Corinth, 12) The American School of Classical Studies at Athens, Princeton. 


